\title{
Dilogarithm Identities for Sine-Gordon and Reduced Sine-Gordon Y-Systems
}

\author{
Tomoki NAKANISHI ${ }^{\dagger}$ and Roberto TATEO $\ddagger$ \\ $\dagger$ Graduate School of Mathematics, Nagoya University, Nagoya, 464-8604, Japan \\ E-mail: nakanisi@math.nagoya-u.ac.jp \\ $\ddagger$ Dipartimento di Fisica Teorica and INFN, Università di Torino, \\ Via P. Giuria 1, 10125 Torino, Italy \\ E-mail: tateo@to.infn.it
}

Received May 29, 2010, in final form October 16, 2010; Published online October 19, 2010 doi:10.3842/SIGMA.2010.085

\begin{abstract}
We study the family of Y-systems and T-systems associated with the sineGordon models and the reduced sine-Gordon models for the parameter of continued fractions with two terms. We formulate these systems by cluster algebras, which turn out to be of finite type, and prove their periodicities and the associated dilogarithm identities which have been conjectured earlier. In particular, this provides new examples of periodicities of seeds.
\end{abstract}

Key words: cluster algebras; quantum groups; integrable models

2010 Mathematics Subject Classification: 13F60; 17B37

\section{Introduction}

The Y-systems and T-systems appeared in the study of two-dimensional integrable $S$-matrix models and integrable lattice models in 90's. They play central roles to connect these integrable models with conformal field theories [3] through the method called the thermodynamic Bethe ansatz (TBA) (e.g., [18, 2, 34, 36, 20, 21, 23, 30, 24, 32]).

Since the introduction of the cluster algebras by Fomin and Zelevinsky [7, 8], it has been gradually recognized that cluster algebras provide a suitable framework to study the mathematical properties of Y and T-systems. As a fruitful outcome, the long standing conjectures of the periodicity of Y-systems [36, 30, 24] (as well as the periodicity of T-systems) and the dilogarithm identities $[19,2,22,12]$ for the class of $\mathrm{Y}$ and T-systems associated with the quantum affine algebras have been proved partly by $[9,5]$, and in full generality by $[16,15,26,17,13,14]$, recently.

In the above proof, the periodicities of the $\mathrm{Y}$ and T-systems are reformulated as the periodicities of seeds in the corresponding cluster algebras. It was shown in [13] that the periodicity of seeds in a cluster algebra associated with a skew symmetric matrix reduces to the periodicity of the tropical coefficients (the 'principal coefficients' in [10]), which is much simpler than the original problem. In the above examples, the periodicities at the level of the tropical coefficients are realized as combinations of the Coxeter transformations of the $A-D-E$ root systems and their variations. From this point of view, one can regard these periodicities as a natural extension of the periodicities of the Coxeter mutation sequences in the cluster algebras of finite type studied by $[8,9,10]$. Then, it is natural to ask the following question: "Are there any other periodicities of seeds? And, if there are, can we classify them?"

The purpose of this paper is to present a new class of (infinitely many) periodicities of seeds in cluster algebras. These cluster algebras correspond to the Y-systems introduced in [32], where their periodicities and the associated dilogarithm identities were also conjectured. More 


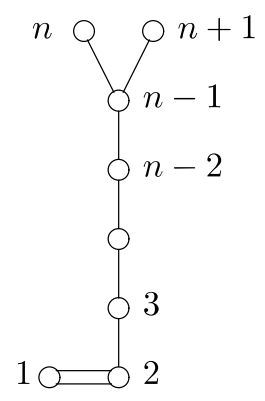

Figure 1. The diagram $X_{n}$.

precisely, these Y-systems consist of two classes. The first class are called the sine-Gordon (SG) $Y$-systems and they are associated with the TBA equation for the sine-Gordon model [35]. The second class are called the reduced sine-Gordon $(R S G)$ Y-systems and, as the name suggests, they are associated with the TBA equation for a certain reduction of the SG model [31, 4]. To these cluster algebras, one can apply the method of [13], and prove their periodicities and the associated dilogarithm identities. This is the main result of the paper. To be more precise, we concentrate on the case where the 'coupling constant' $\xi$ is a continued fraction with two terms, for simplicity.

The result also suggests us a vague perspective to the second question above. Namely, the classification of the periodicities of seeds may be comparable with the classification of the integrable deformations of rational conformal field theories. In particular, those periodicities we have already known should be just a tip of iceberg.

The content of the paper is as follows. In Section 2 we introduce the Y-systems and T-systems, then summarize the results of their periodicities and the associated dilogarithm identities in both the SG and the RSG cases. Here, we concentrate on the special case of the choice of the coupling constant $\xi$ in (2.2). In Sections 3 and 4 we give a proof in the SG case. In Sections 5 and 6 we give a proof in the RSG case. In Section 7 we extend the result to a little more general case of $\xi$ in (7.1), i.e., a general continued fraction with two terms.

In the derivation of the main result, the properties of the tropical Y-systems in Propositions 4.2 and 6.1 are crucial, and the proofs are provided in detail. The proofs of the rest are rather formal and repetitions of the formerly studied cases [16, 15, 26, 17, 13, 14]. So, instead of repeating similar proofs, we only provide examples which typically representing situations.

Note added. After the submission of the paper, the anonymous referee pointed out us that the cluster algebras concerned with the SG and RSG models studied in this paper turn out to be the cluster algebras of type $D$ and $A$, respectively. Since this is a very important fact, we include it in a new subsection (Section 7.3). This means, somewhat on the contrary to our initial intention and expectation, the periodicities of the mutation sequences studied in this paper are actually the ones inside the cluster algebras of finite type. In particular, the periodicity property itself is an automatic consequence of the finiteness of the number of seeds. However, the determination of their precise periods is still new in the literature; furthermore, it does not crucially change our perspective of the richness of the periodicity phenomena of seeds, which should be uncovered in the future study.

\section{Main results}

\subsection{Results for sine-Gordon Y-systems}

With an integer $n \geq 4$, we associate a diagram $X_{n}$ in Fig. 1 . The diagram $X_{n}$ should not be regarded as an ordinary Dynkin diagram, since the horizontality and verticality of segments also 
carry information. It appeared in [32] and encodes the structure of the Y-systems which we are going to introduce now. Let $\mathcal{I}_{n}=\{1, \ldots, n+1\} \times \mathbb{Z}$.

Definition 2.1. Fix an integer $n \geq 4$. The sine-Gordon (SG) Y-system $\mathbb{Y}_{n}(\mathrm{SG})$ is the following system of relations for a family of variables $\left\{Y_{i}(u) \mid(i, u) \in \mathcal{I}_{n}\right\}$,

$$
\begin{aligned}
& Y_{1}(u-n+1) Y_{1}(u+n-1)=\left(\prod_{j=2}^{n-1}\left(1+Y_{j}(u-n+j)\right)\left(1+Y_{j}(u+n-j)\right)\right) \\
& \quad \times\left(1+Y_{n}(u)\right)\left(1+Y_{n+1}(u)\right), \\
& Y_{2}(u-1) Y_{2}(u+1)=\frac{1+Y_{1}(u)}{1+Y_{3}(u)^{-1}}, \\
& Y_{i}(u-1) Y_{i}(u+1)=\frac{1}{\prod_{j: j \sim i}\left(1+Y_{j}(u)^{-1}\right)}, \quad i=3, \ldots, n+1,
\end{aligned}
$$

where $j \sim i$ means that $j$ is adjacent to $i$ in $X_{n}$.

In [32], a more general family of Y-systems was associated with a rational parameter $\xi$, which is related the coupling constant $\beta$ of the SG model by (2.15). The system (2.1) corresponds to the special case

$$
\xi=\frac{n-1}{n}=\frac{1}{1+\frac{1}{n-1}},
$$

namely, $F=2, n_{1}=1$, and $n_{2}=n$ in the notation in [32]. The variable $u$ here is related to the variable $\theta$ in [32] by $u=(2 n / \pi \sqrt{-1}) \theta$. Later in Section 2.3 we explain more about the background of (2.1).

Definition 2.2. Let $y_{n}(\mathrm{SG})$ be the semifield (Appendix A(i)) with generators $Y_{i}(u)\left((i, u) \in \mathcal{I}_{n}\right)$ and relations $\mathbb{Y}_{n}(\mathrm{SG})$. Let $\mathfrak{y}_{n}^{\circ}(\mathrm{SG})$ be the multiplicative subgroup of $y_{n}(\mathrm{SG})$ generated by $Y_{i}(u)$, $1+Y_{i}(u)\left((i, u) \in \mathcal{I}_{n}\right)$. (Here we use the symbol + instead of $\oplus$ for simplicity.)

The first main result of the paper is the following two theorems conjectured by [32].

Theorem 2.3 (Periodicity). The following relations hold in $y_{n}^{\circ}(\mathrm{SG})$.

(i) Half periodicity: $Y_{i}(u+4 n-2)=Y_{\omega(i)}(u)$, where $\omega$ is an involution of the set $\{1, \ldots, n+1\}$ defined by $\omega(n)=n+1, \omega(n+1)=n$, and $\omega(i)=i(i=1, \ldots, n-1)$.

(ii) Full periodicity: $Y_{i}(u+8 n-4)=Y_{i}(u)$.

In our proof of Theorem 2.3 we have a natural interpretation of the half period

$$
4 n-2=h\left(D_{n}\right)+2+h\left(D_{n-1}\right)+2
$$

in terms of the Coxeter number $h\left(D_{n}\right)=2 n-2$ of type $D_{n}$.

Let $L(x)$ be the Rogers dilogarithm function [25]

$$
L(x)=-\frac{1}{2} \int_{0}^{x}\left\{\frac{\log (1-y)}{y}+\frac{\log y}{1-y}\right\} d y, \quad 0 \leq x \leq 1 .
$$

It satisfies the following relation

$$
L(x)+L(1-x)=\frac{\pi^{2}}{6}, \quad 0 \leq x \leq 1 .
$$


Theorem 2.4 (Functional dilogarithm identities). Suppose that a family of positive real numbers $\left\{Y_{i}(u) \mid(i, u) \in \mathcal{I}_{n}\right\}$ satisfies $\mathbb{Y}_{n}(\mathrm{SG})$. Then, we have the identities

$$
\begin{aligned}
& \frac{6}{\pi^{2}} \sum_{\substack{(i, u) \in \mathcal{I}_{n} \\
0 \leq u<8 n-4}} L\left(\frac{Y_{i}(u)}{1+Y_{i}(u)}\right)=8(2 n-1), \\
& \frac{6}{\pi^{2}} \sum_{\substack{(i, u) \in \mathcal{I}_{n} \\
0 \leq u<8 n-4}} L\left(\frac{1}{1+Y_{i}(u)}\right)=4(n-1)(2 n-1) .
\end{aligned}
$$

Two identities (2.4) and (2.5) are equivalent to each other due to (2.3).

Using this opportunity, we introduce another system of relations accompanying $\mathbb{Y}_{n}(\mathrm{SG})$.

Definition 2.5. Fix an integer $n \geq 4$. The sine-Gordon (SG) T-system $\mathbb{T}_{n}(\mathrm{SG})$ is the following system of relations for a family of variables $\left\{T_{i}(u) \mid(i, u) \in \mathcal{I}_{n}\right\}$,

$$
\begin{aligned}
& T_{1}(u-n+1) T_{1}(u+n-1)=T_{2}(u)+1, \\
& T_{2}(u-1) T_{2}(u+1)=T_{1}(u-n+2) T_{1}(u+n-2)+T_{3}(u), \\
& T_{i}(u-1) T_{i}(u+1)=T_{1}(u-n+i) T_{1}(u+n-i)+\prod_{j: j \sim i} T_{j}(u), \quad i=3, \ldots, n-1, \\
& T_{n}(u-1) T_{n}(u+1)=T_{1}(u)+T_{n-1}(u), \\
& T_{n+1}(u-1) T_{n+1}(u+1)=T_{1}(u)+T_{n-1}(u),
\end{aligned}
$$

where $j \sim i$ means that $j$ is adjacent to $i$ in $X_{n}$.

There are two connections between $\mathbb{Y}_{n}(\mathrm{SG})$ and $\mathbb{T}_{n}(\mathrm{SG})$. The first connection is a formal one. Set

$$
d_{1}=n-1, \quad d_{i}=1, \quad i=2, \ldots, n-1,
$$

and let us write (2.1) in a unified manner as

$$
Y_{i}\left(u-d_{i}\right) Y_{i}\left(u+d_{i}\right)=\frac{\prod_{(j, v) \in \mathcal{I}_{n}}\left(1+Y_{j}(v)\right)^{G_{+}(j, v ; i, u)}}{\prod_{(j, v) \in \mathcal{I}_{n}}\left(1+Y_{j}(v)^{-1}\right)^{G_{-}(j, v ; i, u)}} .
$$

Then, (2.6) is written as

$$
T_{i}\left(u-d_{i}\right) T_{i}\left(u+d_{i}\right)=\prod_{(j, v) \in \mathcal{I}_{n}} T_{j}(v)^{G_{+}(i, u ; j, v)}+\prod_{(j, v) \in \mathcal{I}_{n}} T_{j}(v)^{G_{-}(i, u ; j, v)} .
$$

Note that we took the 'transpositions' of $G_{+}$and $G_{-}$in (2.9). The second connection is an algebraic one. Suppose that $\left\{T_{i}(u) \mid(i, u) \in \mathcal{I}_{n}\right\}$ satisfies the T-system $\mathbb{T}_{n}(\mathrm{SG})$. Set

$$
Y_{i}(u)=\frac{\prod_{(j, v) \in \mathcal{I}_{n}} T_{j}(v)^{G_{+}(i, u ; j, v)}}{\prod_{(j, v) \in \mathcal{I}_{n}} T_{j}(v)^{G_{-}(i, u ; j, v)}} .
$$

Then, $\left\{Y_{i}(u) \mid(i, u) \in \mathcal{I}_{n}\right\}$ satisfies the $\mathrm{Y}$-system $\mathbb{Y}_{n}(\mathrm{SG})$. One may check the claim directly at this moment using $\mathbb{T}_{n}(\mathrm{SG})$ (with some effort). Alternatively and better, one can automatically obtain it from [10, Proposition 3.9] once we formulate these systems by a cluster algebra in the next section. 
Definition 2.6. Let $\mathcal{T}_{n}(\mathrm{SG})$ be the commutative ring over $\mathbb{Z}$ with identity element, with generators $T_{i}(u)^{ \pm 1}\left((i, u) \in \mathcal{I}_{n}\right)$ and relations $\mathbb{T}_{n}(\mathrm{SG})$ together with $T_{i}(u) T_{i}(u)^{-1}=1$. Let $\mathcal{T}_{n}^{\circ}(\mathrm{SG})$ be the subring of $\mathcal{T}_{n}(\mathrm{SG})$ generated by $T_{i}(u)\left((i, u) \in \mathcal{I}_{n}\right)$.

The following theorem can be proved simultaneously with Theorem 2.3.

Theorem 2.7 (Periodicity). The following relations hold in $\mathcal{T}_{n}^{\circ}(\mathrm{SG})$.

(i) Half periodicity: $T_{i}(u+4 n-2)=T_{\omega(i)}(u)$, where $\omega$ is the one in Theorem 2.3.

(ii) Full periodicity: $T_{i}(u+8 n-4)=T_{i}(u)$.

Remark 2.8. Actually, $\mathbb{Y}_{n}(\mathrm{SG})$ and $\mathbb{T}_{n}(\mathrm{SG})$ are also considered for $n=3$, and they coincide with the $\mathrm{Y}$ and T-systems of type $B_{2}$ with level 2 in [24]. Theorems 2.3, 2.4, and 2.7 remain valid for $n=3$ due to [13].

All the results in this subsection will be extended to a more general case (7.1) later in Section 7 .

\subsection{Results for reduced sine-Gordon Y-systems}

The SG Y-system in the previous subsection admits a reduction called the reduced SG Y-system. It is obtained from (2.1) by formally setting $Y_{n}(u), Y_{n+1}(u) \rightarrow-1$ and $Y_{n-1}(u) \rightarrow \infty$ [32]. Let $\tilde{\mathcal{I}}_{n}=\{1, \ldots, n-2\} \times \mathbb{Z}$.

Definition 2.9. Fix an integer $n \geq 4$. The reduced sine-Gordon (RSG) Y-system $\mathbb{Y}_{n}(\mathrm{RSG})$ is the following system of relations for a family of variables $\left\{Y_{i}(u) \mid(i, u) \in \tilde{\mathcal{I}}_{n}\right\}$,

$$
\begin{aligned}
& Y_{1}(u-n+1) Y_{1}(u+n-1)=\frac{\prod_{j=2}^{n-2}\left(1+Y_{j}(u-n+j)\right)\left(1+Y_{j}(u+n-j)\right)}{1+Y_{n-2}(u)^{-1}}, \\
& Y_{2}(u-1) Y_{2}(u+1)=\frac{1+Y_{1}(u)}{1+Y_{3}(u)^{-1}}, \\
& Y_{i}(u-1) Y_{i}(u+1)=\frac{1}{\prod_{j: j \sim i}\left(1+Y_{j}(u)^{-1}\right)}, \quad i=3, \ldots, n-2,
\end{aligned}
$$

where $j \sim i$ means that $j \leq n-2$ is adjacent to $i$ in $X_{n}$. For $n=4$, the second relation is replaced with

$$
Y_{2}(u-1) Y_{2}(u+1)=1+Y_{1}(u) .
$$

Definition 2.10. Let $y_{n}(\mathrm{RSG})$ be the semifield with generators $Y_{i}(u)\left((i, u) \in \tilde{\mathcal{I}}_{n}\right)$ and relations $\mathbb{Y}_{n}(\mathrm{RSG})$. Let $\mathrm{y}_{n}^{\circ}(\mathrm{RSG})$ be the multiplicative subgroup of $y_{n}(\mathrm{RSG})$ generated by $Y_{i}(u), 1+Y_{i}(u)$ $\left((i, u) \in \tilde{\mathcal{I}}_{n}\right)$.

The second main result of the paper is the following two theorems conjectured by [32]. The first theorem was already proved by [12] for the RSG Y-systems associated with a general rational $\xi$ by using the explicit solution in terms of cross-ratio.

Theorem 2.11 (Periodicity [12]). The following relations hold in $y_{n}^{\circ}(\mathrm{RSG})$.

Periodicity: $Y_{i}(u+4 n-2)=Y_{i}(u)$.

In our proof of Theorem 2.11 we have a natural interpretation of the period

$$
4 n-2=2\left\{h\left(A_{n-3}\right)+2+h\left(A_{n-4}\right)+2\right\}
$$

in terms of the Coxeter number $h\left(A_{n}\right)=n+1$ of type $A_{n}$. 
Theorem 2.12 (Functional dilogarithm identities). Suppose that a family of positive real numbers $\left\{Y_{i}(u) \mid(i, u) \in \tilde{\mathcal{I}}_{n}\right\}$ satisfies $\mathbb{Y}_{n}(\mathrm{RSG})$. Then, we have the identities

$$
\begin{aligned}
& \frac{6}{\pi^{2}} \sum_{\substack{(i, u) \in \tilde{\mathcal{I}}_{n} \\
0 \leq u<4 n-2}} L\left(\frac{Y_{i}(u)}{1+Y_{i}(u)}\right)=6(2 n-5), \\
& \frac{6}{\pi^{2}} \sum_{\substack{(i, u) \in \tilde{\mathcal{I}}_{n} \\
0 \leq u<4 n-2}} L\left(\frac{1}{1+Y_{i}(u)}\right)=2\left(2 n^{2}-11 n+17\right) .
\end{aligned}
$$

Two identities (2.11) and (2.12) are equivalent to each other due to (2.3).

Again, we introduce the 'T-system' accompanying $\mathbb{Y}_{n}(\mathrm{RSG})$.

Definition 2.13. Fix an integer $n \geq 4$. The reduced sine-Gordon (RSG) T-system $\mathbb{T}_{n}(\mathrm{RSG})$ is the following system of relations for a family of variables $\left\{T_{i}(u) \mid(i, u) \in \tilde{\mathcal{I}}_{n}\right\}$,

$$
\begin{aligned}
& T_{1}(u-n+1) T_{1}(u+n-1)=T_{2}(u)+1, \\
& T_{2}(u-1) T_{2}(u+1)=T_{1}(u-n+2) T_{1}(u+n-2)+T_{3}(u), \\
& T_{i}(u-1) T_{i}(u+1)=T_{1}(u-n+i) T_{1}(u+n-i)+T_{i-1}(u) T_{i+1}(u), \quad i=3, \ldots, n-3, \\
& T_{n-2}(u-1) T_{n-2}(u+1)=T_{1}(u-2) T_{1}(u+2)+T_{1}(u) T_{n-3}(u) .
\end{aligned}
$$

For $n=4$, the second and the fourth relations are replaced with

$$
T_{2}(u-1) T_{2}(u+1)=T_{1}(u-2) T_{1}(u+2)+T_{1}(u) .
$$

There are connections between $\mathbb{Y}_{n}(\mathrm{RSG})$ and $\mathbb{T}_{n}(\mathrm{RSG})$ parallel to the ones between $\mathbb{Y}_{n}(\mathrm{SG})$ and $\mathbb{T}_{n}(\mathrm{SG})$.

Definition 2.14. Let $\mathcal{T}_{n}(\mathrm{RSG})$ be the commutative ring over $\mathbb{Z}$ with identity element, with generators $T_{i}(u)^{ \pm 1}\left((i, u) \in \tilde{\mathcal{I}}_{n}\right)$ and relations $\mathbb{T}_{n}(\mathrm{RSG})$ together with $T_{i}(u) T_{i}(u)^{-1}=1$. Let $\mathcal{T}_{n}^{\circ}(\mathrm{RSG})$ be the subring of $\mathcal{T}_{n}(\mathrm{RSG})$ generated by $T_{i}(u)\left((i, u) \in \tilde{\mathcal{I}}_{n}\right)$.

The following theorem can be proved simultaneously with Theorem 2.11.

Theorem 2.15 (Periodicity). The following relations hold in $\mathcal{T}_{n}^{\circ}(\mathrm{RSG})$.

Periodicity: $T_{i}(u+4 n-2)=T_{i}(u)$.

All the results in this subsection will be extended to a more general case (7.1) later in Section 7.

\subsection{Background in integrable models}

To provide the reader with the 'big picture behind the scene', we briefly review the origins and the consequences of the Y-systems (Definitions 2.1 and 2.9), the periodicity (Theorems 2.3 and 2.11), and the dilogarithm identities (Theorems 2.4 and 2.12) in the context of integrable models. Mathematically speaking, the whole content in this subsection is completely independent of the rest of the paper, so that the reader can safely skip it.

The study of integrable models of quantum field theory has a long history, with two initially distinct lines of development. One comes through classical statistical mechanics, since any lattice model can be viewed as a regularized Euclidean quantum field theory; the other is the direct study of the models as quantum field theories, either in Euclidean or Minkowski space.

On the statistical-mechanical side, the subject can be traced back to Onsager's solution of the two-dimensional Ising model [27] but, much of the modern approach to these models, owes most to the work of Baxter summarized in his book [1]. 
Viewed directly as a problem in quantum field theory, the history begins with the work on the quantum sine-Gordon model, probably the most famous example of an integrable quantum field theory. The Euclidean action of the sine-Gordon model is:

$$
A_{\mathrm{SG}}=\int d x^{2}\left(\frac{1}{16 \pi}\left(\partial_{\nu} \varphi\right)^{2}-2 \mu \cos (\beta \varphi)\right)
$$

where $0<\beta^{2}<1$ is related to $\xi$ in $(2.2)$ as

$$
\xi=\frac{\beta^{2}}{1-\beta^{2}},
$$

and $\mu$ fixes the mass scale: $m \propto \mu^{1 /\left(2-2 \beta^{2}\right)}$, where $m$ is the soliton mass. For $\xi<1$ the theory comprises also solitons-antisoliton bound states - the breathers - with masses

$$
m_{n}=2 m \sin (\pi n \xi / 2), \quad n=1,2, \ldots<1 / \xi .
$$

The review by Zamolodchikov and Zamolodchikov [35] covers most of this early work, which focused particularly on characteristics of the models when defined in infinite spatial volumes.

The main goal when studying any theory was the exact calculation of its $S$-matrix, describing the scattering of arbitrary numbers of elementary excitations.

All of this work concerned massive quantum field theories, with finite correlation lengths. By contrast, the initial interest in statistical mechanics is often the study of models at criticality, where the correlation length is infinite.

Links between the two approaches began to be built with the development of conformal field theory (CFT) [3] which showed how powerful algebraic techniques could be used to solve massless quantum field theories, corresponding to the continuum limits of critical lattice models.

Most relevant to the current context was the subsequent discovery by Zamolodchikov [33] that suitable perturbations, of these conformal field theories could lead to models of precisely the sorts which had been studied previously as massive integrable quantum field theories and exact $S$-matrix models. The Euclidean action of a perturbed CFT is

$$
A_{\mu}=A_{\mathrm{CFT}}+\mu \int d x^{2} \phi(x),
$$

where $A_{\mathrm{CFT}}$ is the action of the conformal invariant theory and $\phi$ is a spinless primary field with conformal dimensions $\Delta=\bar{\Delta}<1$. The dimensionful coupling $\mu$ measures the deviation from the critical point and introduces an independent mass scale proportional to $\mu^{1 /(2-2 \Delta)}$. Comparing (2.14) with (2.16), we see that the sine-Gordon model corresponds to the perturbation of a CFT with central charge $c=1$ - a free massless boson - by the operator $\phi_{\mathrm{SG}}=2 \cos (\beta \varphi)$ with conformal dimension $\Delta_{\mathrm{SG}}=\beta^{2}$.

Another interesting family of exact $S$-matrix models is obtained from the sine-Gordon model, at rational values of $\beta^{2}$, through a quantum group restriction [31, 4] of the Hilbert space.

Setting $\beta^{2}=p / q$ with $q>p$ coprime integers, this infinite family of models corresponds to the minimal conformal field theories $\mathcal{M}_{p, q}$ perturbed by the operator $\phi_{1,3}$ with $\Delta_{\mathrm{RSG}}=(2 p-q) / q$.

As we will see shortly, it is not the Virasoro central charge $c=1-6(p-q)^{2} / p q$ but the effective central charge $c_{\text {eff }}=c-24 \Delta_{0}$, with $\Delta_{0}$ the dimension of the field $\phi_{0}$, which generates the ground state of the conformal field theory on a cylinder. For the $\mathcal{M}_{p, q}$ family of models $c_{\text {eff }}=1-6 / p q$.

Links with statistical mechanics grew stronger as finite-size effects began to be explored, using techniques such as the thermodynamic Bethe Ansatz (TBA) [34]. In particular, sets of functional relations, the Y-systems, began to emerge [36] which very closely paralleled mathematical structures such as the 'fusion hierarchies' (T-systems) found in the developments of Baxter's pioneering work on integrable lattice models [24]. 
In the TBA approach, the ground state energy $E_{0}(R)$ of a massive integrable quantum field theory confined on an infinitely long cylinder of circumference $R$ is written in terms of dressed single-particle energies $\varepsilon_{a}(\theta)$ (pseudoenergies) as

$$
E_{0}(R)=-\frac{\pi c_{\mathrm{eff}}(r)}{6 R}=-\frac{1}{2 \pi} \sum_{i=1}^{N} \int_{-\infty}^{\infty} d \theta \nu_{i}(\theta) \ln \left(1+e^{-\varepsilon_{i}(\theta)}\right),
$$

where $\theta$ is the rapidity and $r$ is related to the mass $m_{1}$ of the lightest excitation in the theory and the circumference $R$ by $r=R m_{1}$. The pseudoenergies are the solutions of a set of coupled integral equations known as TBA equations. The latter equations have the general form

$$
\varepsilon_{i}(\theta)=\nu_{i}(\theta)-\sum_{j=1}^{N} \int_{-\infty}^{\infty} d \theta \phi_{i j}\left(\theta-\theta^{\prime}\right) \ln \left(1+e^{-\varepsilon_{j}\left(\theta^{\prime}\right)}\right) .
$$

When all scattering is diagonal, the integral equations of the TBA follow directly from the mass spectrum $\left\{m_{i}\right\}$ and the two-body $S$-matrix elements $S_{i j}$ :

$$
\nu_{i}(\theta)=R m_{i} \cosh \theta, \quad \phi_{i j}(\theta)=\frac{1}{2 \pi \sqrt{-1}} \frac{d}{d \theta} \ln S_{i j}(\theta) .
$$

In this case, the number of pseudoenergies coincides with the number $\mathrm{N}$ of particle types in the original scattering theory. If the scattering is non-diagonal the TBA derivation becomes more complicated but the final result can be still written in the form (2.18), with some of the $\nu_{i}=0$. The pseudoenergies with $\nu_{i}=0$ correspond to fictitious particles transporting zero energy and zero momentum. These new particles are often called 'magnons', and can be thought of as constructs introduced to get the counting of states right. The Y functions, the main subject of this paper, are related to the pseudoenergies as $Y_{i}(\theta)=e^{\varepsilon_{i}(\theta)}$.

The ultraviolet CFT regime corresponds to $m_{1} \rightarrow 0$ or, equivalently, to $r \rightarrow 0$. In this special limit

$$
E_{0}(R) \sim-\frac{\pi c_{\text {eff }}}{6 R}
$$

where $c_{\text {eff }}=c_{\text {eff }}(0)$ is the effective central charge.

It is during the calculation of $c_{\text {eff }}$ from equations (2.17) and (2.18) that sum-rules for the Rogers dilogarithm function emerge:

$$
c_{\text {eff }}=\frac{6}{\pi^{2}} \sum_{i=1}^{N}\left[L\left(\frac{1}{1+Y_{i}}\right)-L\left(\frac{1}{1+\Upsilon_{i}}\right)\right] .
$$

In (2.19), the constants $Y_{i}$ and $\Upsilon_{i}$ are the stationary values of $Y_{i}(\theta)$ in the limits $r \rightarrow 0$ and $r \rightarrow \infty$, respectively.

The TBA equations contain also exact information on the conformal dimension $\Delta$ of the perturbing operator $\phi$. The key idea is to find a set of functional relations satisfied by the Y functions: a Y-system. These relations generally imply a periodicity property for the pseudoenergies under a certain imaginary shift in $\theta$ :

$$
Y_{i}(\theta+2 \pi \sqrt{-1} P)=Y_{i}(\theta)
$$

The periodicity phenomenon was first noticed by Al.B. Zamolodchikov in [36] and considerations, based on this periodicity suggest that in the far ultraviolet region $c_{\text {eff }}(r)$ will have, apart for a possible irregular anti-bulk term, an expansion in powers of $r^{2 / P}$. This implies for $\phi$ either the 
conformal dimension $\Delta=1-1 / P$ and an expansion for $c_{\text {eff }}(r)$ with both even and odd powers of $\mu$, or $\Delta=1-1 / 2 P$ and an expansion where only even powers of $\mu$ appear.

Let us conclude this subsection by demonstrating the validity of the argument for the SG and the RSG models at the simplest case $n=4$ in (2.2). This corresponds to $\xi=3 / 4$ and $\beta^{2}=p / q=3 / 7$. At this specific value of coupling, the sine-Gordon scattering is non-diagonal with a single soliton-antisoliton bound state. Setting $u=8 \theta / \pi \sqrt{-1}$, the corresponding Ysystem is

$$
\begin{aligned}
Y_{1}(u-3) Y_{1}(u+3)= & \left(1+Y_{5}(u)\right)\left(1+Y_{4}(u)\right)\left(1+Y_{3}(u-1)\right) \\
& \times\left(1+Y_{3}(u+1)\right)\left(1+Y_{2}(u-2)\right)\left(1+Y_{2}(u+2)\right), \\
Y_{2}(u-1) Y_{2}(u+1)= & \left(1+Y_{1}(u)\right) \frac{1}{1+Y_{3}(u)^{-1}}, \\
Y_{3}(u-1) Y_{3}(u+1)= & \frac{1}{1+Y_{2}(u)^{-1}} \frac{1}{1+Y_{4}(u)^{-1}} \frac{1}{1+Y_{5}(u)^{-1}}, \\
Y_{4}(u-1) Y_{4}(u+1)= & \frac{1}{1+Y_{3}(u)^{-1}}, \quad Y_{5}(u-1) Y_{5}(u+1)=\frac{1}{1+Y_{3}(u)^{-1}} .
\end{aligned}
$$

In (2.20), $Y_{1}$ corresponds to the breather, $Y_{2}$ to the soliton-antisoliton pair, $Y_{3}, Y_{4}$ and $Y_{5}$ to magnons. From the point of view of the sine-Gordon ground state energy the two magnonic nodes $Y_{4}$ and $Y_{5}$ are indistinguishable. Thus, it is the half periodicity property (Theorem 2.3), 14 in $u$, and $P=7 / 8$ in $\theta$, that is relevant to our purposes. Considering also that the perturbing field $\phi_{\mathrm{SG}}$ is intrinsically 'self-dual' with only even powers of $\mu$ contributing to the expansion of $c_{\text {eff }}(r)$, the final result is $\Delta=1-1 / 2 P=3 / 7$. This coincides with the conformal dimension of $\phi_{\mathrm{SG}}$.

For the dilogarithm sum-rule (2.19) at $r \rightarrow 0$, instead of solving the stationary version of the Y-system we can simply use the result (2.5) averaged over the period:

$$
\frac{6}{\pi^{2}} \sum_{i=1}^{n+1} L\left(\frac{1}{1+Y_{i}}\right)=\frac{1}{8 n-4} \frac{6}{\pi^{2}} \sum_{\substack{(i, u) \in \mathcal{I}_{n} \\ 0 \leq u<8 n-4}} L\left(\frac{1}{1+Y_{i}(u)}\right)=n-1,
$$

with $n=4$. Similarly, in the limit $r \rightarrow \infty$ both $Y_{1}(u)$ and $Y_{2}(u)$ tend to infinity and decouple. Hence, for the second contribution to the sum-rule (2.19) we can consider the simplified system

$$
\begin{aligned}
& \Upsilon_{3}(u-1) \Upsilon_{3}(u+1)=\frac{1}{1+\Upsilon_{4}(u)^{-1}} \frac{1}{1+\Upsilon_{5}(u)^{-1}}, \\
& \Upsilon_{4}(u-1) \Upsilon_{4}(u+1)=\frac{1}{1+\Upsilon_{3}(u)^{-1}}, \quad \Upsilon_{5}(u-1) \Upsilon_{5}(u+1)=\frac{1}{1+\Upsilon_{3}(u)^{-1}} .
\end{aligned}
$$

The latter is a $D_{3} \equiv A_{3}$ Y-system. Due to [12], the half-period is 6 in the variable $u$, and we have

$$
\frac{6}{\pi^{2}} \sum_{i=3}^{5} L\left(\frac{1}{1+\Upsilon_{i}}\right)=\frac{1}{\pi^{2}} \sum_{\substack{(i, u) \in D_{3} \\ 0 \leq u<6}} L\left(\frac{1}{1+\Upsilon_{i}(u)}\right)=2 .
$$

The result is $c_{\text {eff }}=1$, as expected.

The Y-system for the corresponding RSG model is

$$
\begin{aligned}
& Y_{1}(u-3) Y_{1}(u+3)=\left(1+Y_{2}(u-2)\right)\left(1+Y_{2}(u+2)\right) \frac{1}{1+Y_{2}(u)^{-1}}, \\
& Y_{2}(u-1) Y_{2}(u+1)=1+Y_{1}(u) .
\end{aligned}
$$


We still have $P=7 / 8$ (Theorem 2.11) but now the perturbing operator is 'anti self-dual' with respect to the ground state of the conformal field theory on an cylinder. This means that both even and odd powers of $\mu$ appear in the expansion of $c_{\text {eff }}(r)$.

Therefore, $\Delta=1-1 / P=-1 / 7$, which indeed matches the conformal dimension of $\phi_{13}$ in $\mathcal{M}_{3,7}$. Finally, from the result (2.12) we recover the effective central charge of $\mathcal{M}_{3,7}$ :

$$
c_{\text {eff }}=\frac{3}{7 \pi^{2}} \sum_{\substack{(i, u) \in \tilde{\mathcal{I}}_{4} \\ 0 \leq u<14}} L\left(\frac{1}{1+Y_{i}(u)}\right)=\frac{5}{7} .
$$

\section{Cluster algebras for SG Y-systems}

In this section we identify $\mathbb{T}_{n}(\mathrm{SG})$ and $\mathbb{Y}_{n}(\mathrm{SG})$ as relations for cluster variables and coefficients of the cluster algebra associated with a certain quiver $Q_{n}(\mathrm{SG})$. We follow [10] for definitions and conventions concerning cluster algebras with coefficients, which are summarized in Appendix A for the reader's convenience.

\subsection{Parity decompositions of $\mathrm{T}$ and $\mathrm{Y}$-systems}

For $(i, u) \in \mathcal{I}_{n}$, we set the parity condition $\mathbf{P}_{+}$by, for even $n$,

$$
\mathbf{P}_{+}: \begin{cases}i+u \text { is even, } & i=1, \ldots, n \\ n+u \text { is even, } & i=n+1,\end{cases}
$$

and, for odd $n$,

$$
\mathbf{P}_{+}: \begin{cases}u \text { is even, } & i=1, \\ i+u \text { is even, } & i=2, \ldots, n, \\ n+u \text { is even, } & i=n+1 .\end{cases}
$$

Let $\mathbf{P}_{-}$be the negation of $\mathbf{P}_{+}$. We write, for example, $(i, u): \mathbf{P}_{+}$if $(i, u) \in \mathcal{I}_{n}$ satisfies $\mathbf{P}_{+}$. Let $\mathcal{I}_{n \varepsilon}(\varepsilon= \pm)$ be the set of all $(i, u): \mathbf{P}_{\varepsilon}$. Define $\mathcal{T}_{n}^{\circ}(\mathrm{SG})_{\varepsilon}(\varepsilon= \pm)$ to be the subring of $\mathcal{T}_{n}^{\circ}(\mathrm{SG})$ generated by $T_{i}(u)\left((i, u) \in \mathcal{I}_{n \varepsilon}\right)$. Then, we have $\mathcal{T}_{n}^{\circ}(\mathrm{SG})_{+} \simeq \mathcal{T}_{n}^{\circ}(\mathrm{SG})_{-}$by $T_{i}(u) \mapsto T_{i}(u+1)$ and

$$
\mathcal{T}_{n}^{\circ}(\mathrm{SG}) \simeq \mathcal{T}_{n}^{\circ}(\mathrm{SG})_{+} \otimes_{\mathbb{Z}} \mathcal{T}_{n}^{\circ}(\mathrm{SG})_{-} .
$$

For $(i, u) \in \mathcal{I}_{n}$, we introduce another parity condition $\mathbf{P}_{+}^{\prime}$ by

$$
\mathbf{P}_{+}^{\prime}: \begin{cases}i+u \text { is odd, } & i=1, \ldots, n, \\ n+u \text { is odd, }, & i=n+1,\end{cases}
$$

We have

$$
(i, u): \mathbf{P}_{+}^{\prime} \Longleftrightarrow\left(i, u \pm d_{i}\right): \mathbf{P}_{+},
$$

where $d_{i}$ is given in (2.7). Let $\mathbf{P}_{-}^{\prime}$ be the negation of $\mathbf{P}_{+}^{\prime}$. Let $\mathcal{I}_{n \varepsilon}^{\prime}(\varepsilon= \pm)$ be the set of all $(i, u): \mathbf{P}_{\varepsilon}^{\prime}$. Define $y_{n}^{\circ}(\mathrm{SG})_{\varepsilon}(\varepsilon= \pm)$ to be the subgroup of $y_{n}^{\circ}(\mathrm{SG})$ generated by $Y_{i}(u), 1+Y_{i}(u)$ $\left((i, u) \in \mathcal{I}_{n \varepsilon}^{\prime}\right)$. Then, we have $y_{n}^{\circ}(\mathrm{SG})_{+} \simeq y_{n}^{\circ}(\mathrm{SG})_{-}$by $Y_{i}(u) \mapsto Y_{i}(u+1), 1+Y_{i}(u) \mapsto 1+Y_{i}(u+1)$, and

$$
y_{n}^{\circ}(\mathrm{SG}) \simeq y_{n}^{\circ}(\mathrm{SG})_{+} \times y_{n}^{\circ}(\mathrm{SG})_{-} .
$$

From now on, we mainly treat the + parts, $\mathcal{T}_{n}^{\circ}(\mathrm{SG})_{+}$and $y_{n}^{\circ}(\mathrm{SG})_{+} \cdot$ 

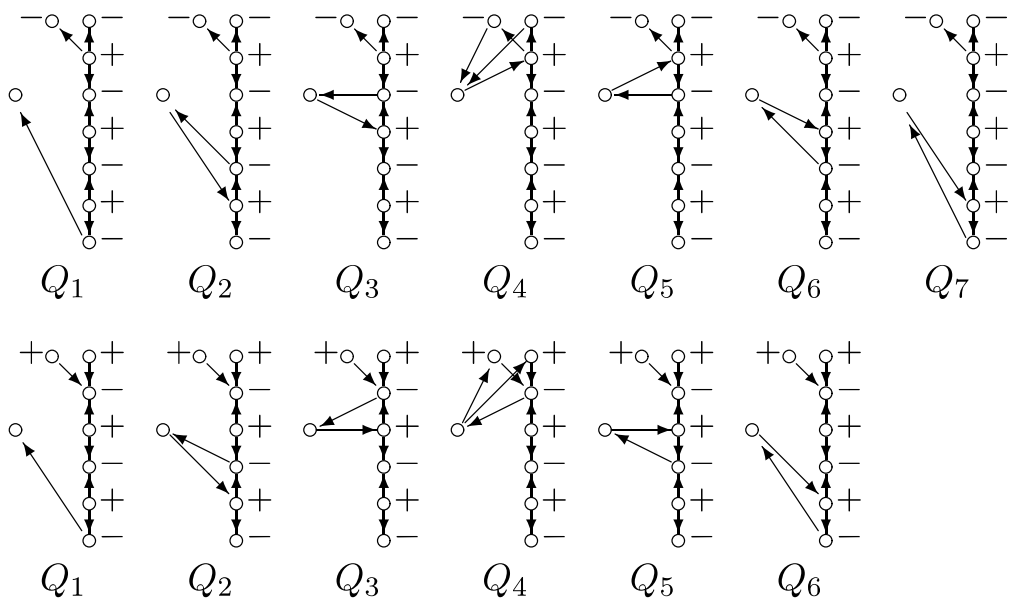

Figure 2. The quiver $Q_{n}(\mathrm{SG})$ for $n=8$ (upper) and for $n=7$ (lower), where, except for the leftmost vertex of each quiver $Q_{i}$, all the vertices in the same position in $n-1$ quivers $Q_{1}, \ldots, Q_{n-1}$ are identified.

\subsection{Quiver $Q_{n}(\mathrm{SG})$}

Recall that a quiver is an oriented graph, namely, it consists of the vertices and the arrows connecting them. With each $n \geq 4$ we associate a quiver $Q_{n}(\mathrm{SG})$ as below. First, as rather general examples, the cases $n=8$ and $n=7$ are given in Fig. 2, where, except for the leftmost vertex of each quiver $Q_{i}$, all the vertices in the same position in $n-1$ quivers $Q_{1}, \ldots, Q_{n-1}$ are identified. For a general $n$, the quiver $Q_{n}(\mathrm{SG})$ is defined by naturally extending these examples. Namely, we consider $n-1$ quivers $Q_{1}, \ldots, Q_{n-1}$, whose vertices are naturally identified with the vertices of the graph $X_{n}$ in Fig. 1 . (The leftmost vertex corresponds to the vertex 1 in $X_{n}$.) The arrows are put in $Q_{i}$ as clearly indicated by the examples in Fig. 2. Note that the pattern of arrows slightly depends on the parity of $n$. Then, except for the leftmost vertex of each quiver $Q_{i}$, all the vertices in the same position in $n-1$ quivers $Q_{1}, \ldots, Q_{n-1}$ are identified. Also we assign the property $+/-$ to each vertex, except for the leftmost one in each $Q_{i}$, as in Fig. 2.

Let us choose the index set $\mathbf{I}$ of the vertices of $Q_{n}(\mathrm{SG})$ so that $\mathbf{i}=(i, 1) \in \mathbf{I}$ represents the leftmost vertex in $Q_{i}$ for $i=1, \ldots, n-1$, and $\mathbf{i}=\left(n, i^{\prime}\right) \in \mathbf{I}$ represents the vertex $i^{\prime}=2, \ldots, n+1$ in any quiver $Q_{i}$ under the natural identification with $X_{n}$. Thus, $i=1, \ldots, n$; and $i^{\prime}=1$ if $i \neq n$ and $i^{\prime}=2, \ldots, n+1$ if $i=n$.

Let $\mathbf{I}_{+}$(resp. $\mathbf{I}_{-}$) denote the set of the vertices $\mathbf{i} \in \mathbf{I}$ with property + (resp. - ). We define composite mutations (Appendix A(ii)-(v)),

$$
\mu_{+}=\prod_{\mathbf{i} \in \mathbf{I}_{+}} \mu_{\mathbf{i}}, \quad \mu_{-}=\prod_{\mathbf{i} \in \mathbf{I}_{-}} \mu_{\mathbf{i}}
$$

Note that they do not depend on the order of the product.

For a permutation $\sigma$ of $\{1, \ldots, n-1\}$, let $\tilde{\sigma}$ be the permutation of $\mathbf{I}$ such that $\tilde{\sigma}(i, 1)=(\sigma(i), 1)$ for $i \neq n$ and $\tilde{\sigma}\left(n, i^{\prime}\right)=\left(n, i^{\prime}\right)$. Let $\tilde{\sigma}\left(Q_{n}(\mathrm{SG})\right)$ denote the quiver induced from $Q_{n}(\mathrm{SG})$ by $\tilde{\sigma}$. Namely, if there is an arrow $\mathbf{i} \rightarrow \mathbf{j}$ in $Q_{n}(\mathrm{SG})$, then, there is an arrow $\tilde{\sigma}(\mathbf{i}) \rightarrow \tilde{\sigma}(\mathbf{j})$ in $\tilde{\sigma}\left(Q_{n}(\mathrm{SG})\right)$.

Lemma 3.1. Let $Q(0):=Q_{n}(\mathrm{SG})$. We have the following periodic sequence of mutations of quivers:

$$
\begin{aligned}
& Q(0) \stackrel{\mu_{+} \mu_{(1,1)}}{\longleftrightarrow} Q(1) \stackrel{\mu_{-}}{\longleftrightarrow} Q(2) \stackrel{\mu_{+} \mu_{(2,1)}}{\longleftrightarrow} Q(3) \stackrel{\mu_{-}}{\longleftrightarrow} Q(4) \\
& \stackrel{\mu_{+} \mu_{(3,1)}}{\longleftrightarrow} \ldots \stackrel{\mu_{+} \mu_{(n-1,1)}}{\longleftrightarrow} Q(2 n-3) \stackrel{\mu_{-}}{\longleftrightarrow} Q(2 n-2)=Q(0),
\end{aligned}
$$




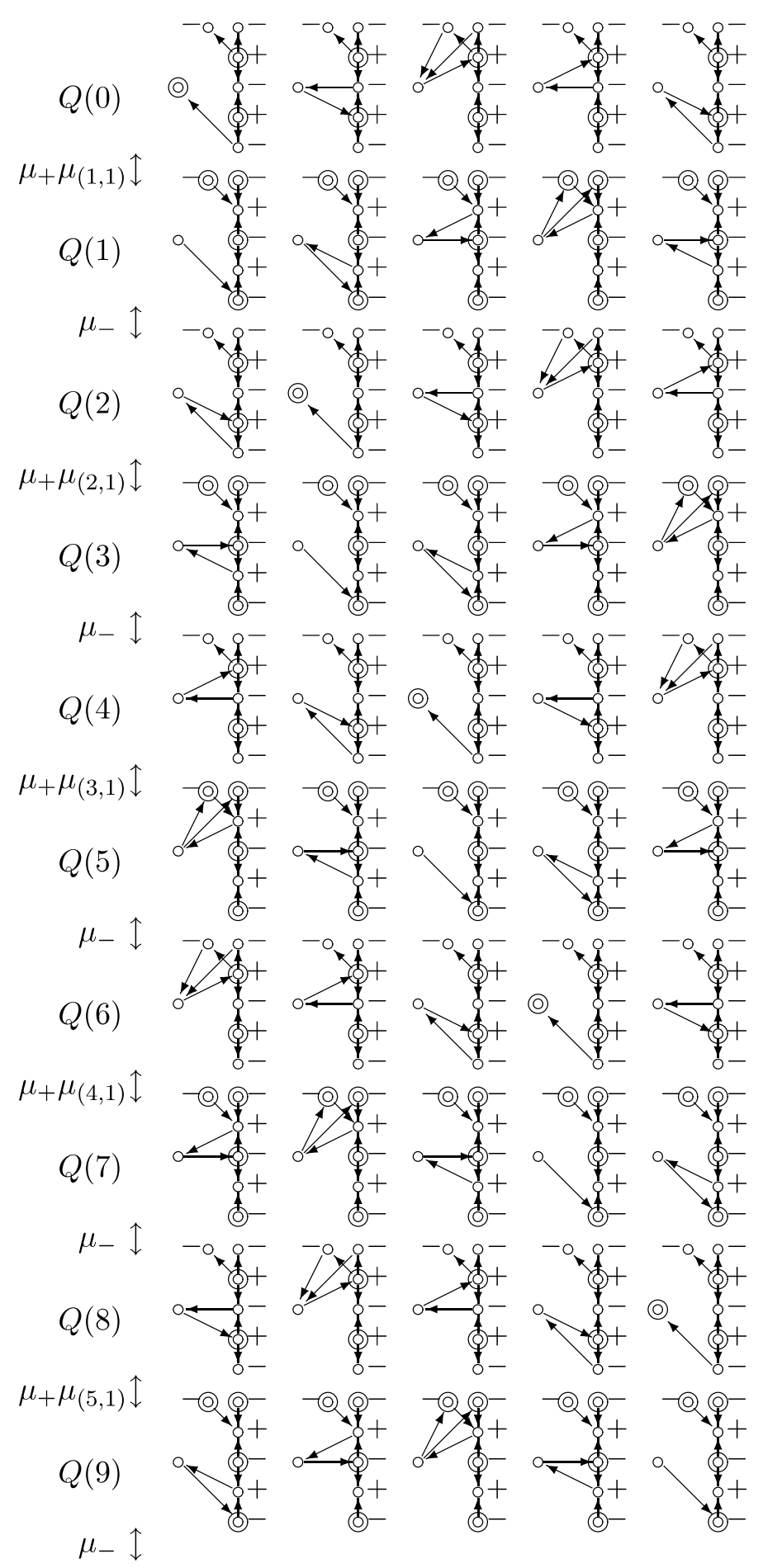

Figure 3. The mutation sequence of the quiver $Q_{n}(\mathrm{SG})$ in (3.1) for $n=6$. The encircled vertices correspond to the mutation points $(\mathbf{i}, u): \mathbf{p}_{+}$in the forward direction.

where the quiver $Q(2 p)(p=1, \ldots, n-2)$ is given by

$$
Q(2 p)=\tilde{\sigma}^{p}(Q(0)), \quad \sigma=\left(\begin{array}{ccccc}
1 & 2 & \ldots & n-2 & n-1 \\
2 & 3 & \ldots & n-1 & 1
\end{array}\right)
$$

Example 3.2. The mutation sequence (3.1) for $n=6$ is explicitly given in Fig. 3. 


\subsection{Embedding maps}

Let $B=B_{n}(\mathrm{SG})$ be the skew-symmetric matrix corresponding to the quiver $Q_{n}(\mathrm{SG})$ (Appendix $\mathrm{A}(\mathrm{iii}))$. Let $\mathcal{A}(B, x, y)$ be the cluster algebra with coefficients in the universal semifield $\mathbb{P}_{\text {univ }}(y)$ (Appendix A(vi)).

In view of Lemma 3.1 we set $x(0)=x, y(0)=y$ and define clusters $x(u)=\left(x_{\mathbf{i}}(u)\right)_{\mathbf{i} \in \mathbf{I}}(u \in \mathbb{Z})$ and coefficient tuples $y(u)=\left(y_{\mathbf{i}}(u)\right)_{\mathbf{i} \in \mathbf{I}}(u \in \mathbb{Z})$ by the sequence of mutations

$$
\begin{aligned}
\cdots & \stackrel{\mu_{-}}{\longleftrightarrow}(B(0), x(0), y(0)) \stackrel{\mu_{+} \mu_{(1,1)}}{\longleftrightarrow}(B(1), x(1), y(1)) \\
& \stackrel{\mu_{-}}{\longleftrightarrow} \ldots \stackrel{\mu_{-}}{\longleftrightarrow}(B(2 n-2), x(2 n-2), y(2 n-2)) \stackrel{\mu_{+} \mu_{(1,1)}}{\longleftrightarrow} \cdots,
\end{aligned}
$$

where $B(u)$ is the skew-symmetric matrix corresponding to $Q(u)$.

For $(\mathbf{i}, u) \in \mathbf{I} \times \mathbb{Z}$, we set the parity condition $\mathbf{p}_{+}$by

$$
\mathbf{p}_{+}: \begin{cases}\mathbf{i} \in \mathbf{I}_{+} \sqcup\{(j+1,1)\}, & u \equiv 2 j(j=0,1, \ldots, n-2), \\ \mathbf{i} \in \mathbf{I}_{-}, & u: \text { odd, }\end{cases}
$$

where $\equiv$ is modulo $(2 n-2) \mathbb{Z}$. We define the condition $\mathbf{p}_{-}$by $(\mathbf{i}, u): \mathbf{p}_{-} \Longleftrightarrow(\mathbf{i}, u-1): \mathbf{p}_{+}$. Plainly speaking, each $(\mathbf{i}, u): \mathbf{p}_{+}$(resp. $\left.(\mathbf{i}, u): \mathbf{p}_{-}\right)$is a mutation point of $(3.3)$ in the forward (resp. backward) direction of $u$. See Fig. 3.

Lemma 3.3. Below $\equiv$ means the equivalence modulo $(2 n-2) \mathbb{Z}$.

(i) The map

$$
\begin{aligned}
g: & \mathcal{I}_{n+} \rightarrow\left\{(\mathbf{i}, u) \in \mathbf{I} \times \mathbb{Z} \mid(\mathbf{i}, u): \mathbf{p}_{+}\right\}, \\
\left(i, u-d_{i}\right) & \mapsto \begin{cases}((j+1,1), u), & i=1 ; u \equiv 2 j(j=0,1, \ldots, n-2), \\
((n, i), u), & i=2, \ldots, n+1\end{cases}
\end{aligned}
$$

is a bijection.

(ii) The map

$$
\begin{aligned}
g^{\prime}: \mathcal{I}_{n+}^{\prime} & \rightarrow\left\{(\mathbf{i}, u) \in \mathbf{I} \times \mathbb{Z} \mid(\mathbf{i}, u): \mathbf{p}_{+}\right\}, \\
(i, u) & \mapsto \begin{cases}((j+1,1), u), & i=1 ; u \equiv 2 j(j=0,1, \ldots, n-2), \\
((n, i), u), & i=2, \ldots, n+1\end{cases}
\end{aligned}
$$

is a bijection.

Based on Lemma 3.3, we introduce alternative notations $\tilde{x}_{i}\left(u-d_{i}\right):=x_{\mathbf{i}}(u)$ for $\left(i, u-d_{i}\right) \in \mathcal{I}_{n+}$ with $(\mathbf{i}, u)=g\left(\left(i, u-d_{i}\right)\right)$ and $y_{i}(u):=y_{\mathbf{i}}(u)$ for $(i, u) \in \mathcal{I}_{n+}^{\prime}$ with $(\mathbf{i}, u)=g^{\prime}((i, u))$, respectively, which turn out to be useful.

Let $\mathcal{A}(B, x)$ be the cluster algebra with trivial coefficients, where $(B, x)$ is the initial seed and the coefficient semifield is the trivial semifield $\mathbf{1}=\{1\}$ (Appendix A(i)). Let $\pi_{\mathbf{1}}: \mathbb{P}_{\text {univ }}(y) \rightarrow \mathbf{1}$, $y_{\mathbf{i}} \mapsto 1$ be the projection. Let $\left[x_{\mathbf{i}}(u)\right]_{\mathbf{1}}$ denote the image of $x_{\mathbf{i}}(u)$ by the algebra homomorphism $\mathcal{A}(B, x, y) \rightarrow \mathcal{A}(B, x)$ induced from $\pi_{\mathbf{1}}$. It is called the trivial evaluation.

The following lemma follows from the exchange relation of cluster variables (A.4) and the property of the sequence (3.1) observed in Fig. 3.

Lemma 3.4. Let $G_{+}$and $G_{-}$be the ones in (2.8) and (2.9). The family $\left\{\tilde{x}_{i}(u) \mid(i, u) \in \mathcal{I}_{n+}\right\}$ satisfies a system of relations

$$
\tilde{x}_{i}\left(u-d_{i}\right) \tilde{x}_{i}\left(u+d_{i}\right)=\frac{y_{i}(u)}{1+y_{i}(u)} \prod_{(j, v) \in \mathcal{I}_{n+}} \tilde{x}_{j}(v)^{G_{+}(i, u ; j, v)}
$$




$$
+\frac{1}{1+y_{i}(u)} \prod_{(j, v) \in \mathcal{I}_{n+}} \tilde{x}_{j}(v)^{G_{-}(i, u ; j, v)}
$$

where $(i, u) \in \mathcal{I}_{n+}^{\prime}$. In particular, the family $\left\{\left[\tilde{x}_{i}(u)\right]_{\mathbf{1}} \mid(i, u) \in \mathcal{I}_{n+}\right\}$ satisfies the T-system $\mathbb{T}_{n}(\mathrm{SG})$ in $\mathcal{A}(B, x)$ by replacing $T_{i}(u)$ with $\left[\tilde{x}_{i}(u)\right]_{\mathbf{1}}$.

Example 3.5. Consider the case $n=6$ in Fig. 3. Let us consider the mutation at the vertex $(1,1)$ in $Q(0)$, to which the variable $\tilde{x}_{1}(-5)$ is attached. The next time $(1,1)$ is mutated is in $Q(10)$, where $\tilde{x}_{1}(5)$ is attached. Meanwhile, the only vertex connected to $(1,1)$ in $Q(0)$ is $(6,2)$, and the variable attached to $(6,2)$ in $Q(0)$ is equal to the variable $\tilde{x}_{2}(0)$ attached to $(6,2)$ in $Q(1)$. Taking account of the directions of the arrows, we have the relation

$$
\tilde{x}_{1}(-5) \tilde{x}_{1}(5)=\frac{y_{1}(0)}{1+y_{1}(0)} \tilde{x}_{2}(0)+\frac{1}{1+y_{1}(0)},
$$

which agrees with (2.6) and (3.5). Similarly, consider the mutation at the vertex $(6,2)$ in $Q(1)$, to which the variable $\tilde{x}_{2}(0)$ is attached. The next time $(6,2)$ is mutated is in $Q(3)$, where $\tilde{x}_{2}(2)$ is attached. Meanwhile, the vertices connected to $(6,2)$ in $Q(1)$ are $(1,1),(2,1)$, and $(6,3)$, and the variable attached to them are equal to $\tilde{x}_{1}(5), \tilde{x}_{1}(-3)$, and $\tilde{x}_{3}(1)$, respectively. Taking account of the directions of the arrows, we have the relation

$$
\tilde{x}_{2}(0) \tilde{x}_{2}(2)=\frac{y_{2}(1)}{1+y_{2}(1)} \tilde{x}_{1}(-3) \tilde{x}_{1}(5)+\frac{1}{1+y_{2}(1)} \tilde{x}_{3}(1) .
$$

As the last example, consider the mutation at the vertex $(6,6)$ in $Q(1)$, to which the variable $\tilde{x}_{6}(0)$ is attached. The next time $(6,6)$ is mutated is in $Q(3)$, where $\tilde{x}_{6}(2)$ is attached. Meanwhile, the vertices connected to $(6,6)$ in $Q(1)$ are $(4,1)$ and $(6,5)$, and the variable attached to them are equal to $\tilde{x}_{1}(1)$ and $\tilde{x}_{5}(1)$, respectively. Taking account of the directions of the arrows, we have the relation

$$
\tilde{x}_{6}(0) \tilde{x}_{6}(2)=\frac{y_{6}(1)}{1+y_{6}(1)} \tilde{x}_{1}(1)+\frac{1}{1+y_{6}(1)} \tilde{x}_{5}(1) .
$$

The other cases can be checked in similar manners.

Definition 3.6. The T-subalgebra $\mathcal{A}_{T}(B, x)$ of $\mathcal{A}(B, x)$ associated with the sequence (3.3) is the subring of $\mathcal{A}(B, x)$ generated by $\left[x_{\mathbf{i}}(u)\right]_{\mathbf{1}}((\mathbf{i}, u) \in \mathbf{I} \times \mathbb{Z})$, or equivalently, generated by $\left[\tilde{x}_{i}(u)\right]_{\mathbf{1}}$ $\left((i, u) \in \mathcal{I}_{n+}\right)$.

By Lemma 3.4, we have the following embedding.

Theorem 3.7. The ring $\mathcal{T}_{n}^{\circ}(\mathrm{SG})_{+}$is isomorphic to $\mathcal{A}_{T}(B, x)$ by the correspondence $T_{i}(u) \mapsto$ $\left[\tilde{x}_{i}(u)\right]_{\mathbf{1}}$.

The coefficient group $\mathcal{G}(B, y)$ associated with $\mathcal{A}(B, x, y)$ is the multiplicative subgroup of the semifield $\mathbb{P}_{\text {univ }}(y)$ generated by all the coefficients $y_{\mathbf{i}}^{\prime}$ of $\mathcal{A}(B, x, y)$ together with $1+y_{\mathbf{i}}^{\prime}$.

The following lemma follows from the exchange relation of coefficients (A.3) and the property of the sequence (3.1).

Lemma 3.8. The family $\left\{y_{i}(u) \mid(i, u) \in \mathcal{I}_{n+}^{\prime}\right\}$ satisfies the $Y$-system $\mathbb{Y}_{n}(\mathrm{SG})$ by replacing $Y_{i}(u)$ with $y_{i}(u)$.

Example 3.9. Consider the case $n=6$ in Fig. 3. Let us consider the mutation at the vertex $(1,1)$ in $Q(0)$, to which the variable $y_{1}(0)$ is attached. The next time $(1,1)$ is mutated is in $Q(10)$, where $y_{1}(10)$ is attached. Meanwhile, between $u=0$ and $u=10$, the vertices connected to $(1,1)$ in $Q(u)$ and mutated are $(6,2)$ at $u=1,9,(6,3)$ at $u=2,8,(6,4)$ at $u=3,7$, 
$(6,5)$ at $u=4,6$, and $(6,6)$ and $(6,7)$ at $u=5$, Taking account of the directions of the arrows, we have the relation

$$
\begin{aligned}
y_{1}(0) y_{1}(10)= & \left(1+y_{2}(1)\right)\left(1+y_{2}(9)\right)\left(1+y_{3}(2)\right)\left(1+y_{3}(8)\right)\left(1+y_{4}(3)\right) \\
& \times\left(1+y_{4}(7)\right)\left(1+y_{5}(4)\right)\left(1+y_{5}(6)\right)\left(1+y_{6}(5)\right)\left(1+y_{7}(5)\right),
\end{aligned}
$$

which agrees with (2.1). Similarly, consider the mutation at the vertex $(6,2)$ in $Q(1)$, to which the variable $y_{2}(1)$ is attached. The next time $(6,2)$ is mutated is in $Q(3)$, where $y_{2}(3)$ is attached. Meanwhile, between $u=1$ and $u=3$, the vertices connected to $(6,2)$ in $Q(u)$ and mutated are $(2,1)$ and $(6,3)$ at $u=2$. Taking account of the directions of the arrows, we have the relation

$$
y_{2}(1) y_{2}(3)=\frac{\left(1+y_{1}(2)\right)}{1+y_{3}(2)^{-1}}
$$

As the last example, consider the mutation at the vertex $(6,6)$ in $Q(1)$, to which the variable $y_{6}(1)$ is attached. The next time $(6,6)$ is mutated is in $Q(3)$, where $y_{6}(3)$ is attached. Meanwhile, between $u=1$ and $u=3$, the only vertex connected to $(6,6)$ in $Q(u)$ and mutated is $(6,5)$ at $u=2$. Taking account of the directions of the arrows, we have the relation

$$
y_{6}(1) y_{6}(3)=\frac{1}{1+y_{5}(2)^{-1}} \text {. }
$$

The other cases can be checked in similar manners.

Definition 3.10. The $Y$-subgroup $\mathcal{G}_{Y}(B, y)$ of $\mathcal{G}(B, y)$ associated with the sequence (3.3) is the subgroup of $\mathcal{G}(B, y)$ generated by $y_{\mathbf{i}}(u)((\mathbf{i}, u) \in \mathbf{I} \times \mathbb{Z})$ and $1+y_{\mathbf{i}}(u)\left((\mathbf{i}, u): \mathbf{p}_{+}\right.$or $\left.\mathbf{p}_{-}\right)$, or equivalently, generated by $y_{i}(u)$ and $1+y_{i}(u)\left((i, u) \in \mathcal{I}_{n+}^{\prime}\right)$.

By Lemma 3.8, we have the following embedding.

Theorem 3.11. The group $y_{n}^{\circ}(\mathrm{SG})_{+}$is isomorphic to $\mathcal{G}_{Y}(B, y)$ by the correspondence $Y_{i}(u) \mapsto$ $y_{i}(u)$ and $1+Y_{i}(u) \mapsto 1+y_{i}(u)$.

\section{Proof of Theorems 2.3, 2.4, and 2.7}

In this section we prove Theorems $2.3,2.4$, and 2.7 using the method of $[26,13]$.

Let $y=y(0)$ be the initial coefficient tuple of the cluster algebra $\mathcal{A}(B, x, y)$ with $B=B_{n}(\mathrm{SG})$ in the previous section. Let $\mathbb{P}_{\text {trop }}(y)$ be the tropical semifield for $y$ (Appendix A(i)). Let $\pi_{\mathbf{T}}: \mathbb{P}_{\text {univ }}(y) \rightarrow \mathbb{P}_{\text {trop }}(y), y_{\mathbf{i}} \mapsto y_{\mathbf{i}}$ be the projection. Let $\left[y_{\mathbf{i}}(u)\right]_{\mathbf{T}}$ and $\left[\mathcal{G}_{Y}(B, y)\right]_{\mathbf{T}}$ denote the images of $y_{\mathbf{i}}(u)$ and $\mathcal{G}_{Y}(B, y)$ by the multiplicative group homomorphism induced from $\pi_{\mathbf{T}}$, respectively. They are called the tropical evaluations, and the resulting relations in the group $\left[\mathcal{G}_{Y}(B, y)\right]_{\mathbf{T}}$ are called the tropical $Y$-system. They are first studied in [9] for cluster algebras of finite types.

We say a (Laurent) monomial $m=\prod_{\mathbf{i} \in \mathbf{I}} y_{\mathbf{i}}^{k_{\mathbf{i}}}$ is positive (resp. negative) if $m \neq 1$ and $k_{\mathbf{i}} \geq 0$ (resp. $k_{\mathbf{i}} \leq 0$ ) for any $\mathbf{i}$. It is known that every monomial $\left[y_{\mathbf{i}}(u)\right]_{\mathbf{T}}$ is either positive or negative by [10, Proposition 5.6] and [6, Theorem 1.7].

The next 'tropical mutation rule' for $\left[y_{\mathbf{i}}(u)\right]_{\mathbf{T}}$ is general and useful.

Lemma 4.1. Suppose that $y^{\prime \prime}$ is the coefficient tuple obtained from the mutation of another coefficient tuple $y^{\prime}$ at $\mathbf{k}$ with mutation matrix $B^{\prime}$. Then, for any $\mathbf{i} \neq \mathbf{k}$, we have the rule:

(i) $\left[y_{\mathbf{i}}^{\prime \prime}\right]_{\mathbf{T}}=\left[y_{\mathbf{i}}^{\prime}\right]_{\mathbf{T}}\left[y_{\mathbf{k}}^{\prime}\right]_{\mathbf{T}}$ if one of the following conditions holds: 
(a) $B_{\mathbf{k i}}^{\prime}>0$, and $\left[y_{\mathbf{k}}^{\prime}\right]_{\mathbf{T}}$ is positive;

(b) $B_{\mathbf{k i}}^{\prime}<0$, and $\left[y_{\mathbf{k}}^{\prime}\right]_{\mathbf{T}}$ is negative.

(ii) $\left[y_{\mathbf{i}}^{\prime \prime}\right]_{\mathbf{T}}=\left[y_{\mathbf{i}}^{\prime}\right]_{\mathbf{T}}$ if one of the following conditions holds:

(a) $B_{\mathbf{k i}}^{\prime}=0$;

(b) $B_{\mathbf{k i}}^{\prime}>0$, and $\left[y_{\mathbf{k}}^{\prime}\right]_{\mathbf{T}}$ is negative;

(c) $B_{\mathbf{k i}}^{\prime}<0$, and $\left[y_{\mathbf{k}}^{\prime}\right]_{\mathbf{T}}$ is positive.

Proof. This is an immediate consequence of the exchange relation (A.3) and (A.1).

The following properties of the tropical Y-system are crucial.

Proposition 4.2. For $\left[\mathcal{G}_{Y}(B, y)\right]_{\mathbf{T}}$ with $B=B_{n}(\mathrm{SG})$, the following facts hold.

(i) For $0 \leq u<4 n-2$, the monomial $\left[y_{\mathbf{i}}(u)\right]_{\mathbf{T}}\left((\mathbf{i}, u): \mathbf{p}_{+}\right)$is negative if and only if $u$ takes the following values.

$$
\begin{cases}2 n-2 \leq u<4 n-2 & \text { for } \mathbf{i}=(1,1), \ldots,(n-1,1),(n, 2), \\ u=2 n-2,2 n-1,4 n-4,4 n-3 & \text { for } \mathbf{i}=(n, 3), \ldots,(n, n+1) .\end{cases}
$$

(Note that for each $\mathbf{i}, u$ takes only a part of the list due to the condition $(\mathbf{i}, u): \mathbf{p}_{+}$.)

(ii) We have $\left[y_{\mathbf{i}}(4 n-2)\right]_{\mathbf{T}}=y_{\tau^{-1}(\mathbf{i})}$, where $\tau$ is a bijection $\mathbf{I} \rightarrow \mathbf{I}$ defined by

$$
\begin{aligned}
& (i, 1) \mapsto(\sigma(i), 1), \quad i=1, \ldots, n-1, \\
& \left(n, i^{\prime}\right) \mapsto \begin{cases}\left(n, i^{\prime}\right), & i^{\prime}=2, \ldots, n-1, \\
(n, n+1), & i^{\prime}=n, \\
(n, n), & i^{\prime}=n+1\end{cases}
\end{aligned}
$$

and $\sigma$ is the permutation in (3.2).

(iii) The number $N_{-}$of the negative monomials $\left[y_{\mathbf{i}}(u)\right]_{\mathbf{T}}$ for $(\mathbf{i}, u): \mathbf{p}_{+}$in the region $0 \leq u<$ $4 n-2$ is $4 n-2$.

Proof. (i) Let us factorize $\left[y_{\mathbf{i}}(u)\right]_{\mathbf{T}}=\left[y_{\mathbf{i}}(u)\right]_{\mathbf{T}}^{\prime}\left[y_{\mathbf{i}}(u)\right]_{\mathbf{T}}^{\prime \prime}$, where $\left[y_{\mathbf{i}}(u)\right]_{\mathbf{T}}^{\prime}$ is a monomial in $y_{(i, 1)}$ $(i=1, \ldots, n-1)$ while $\left[y_{\mathbf{i}}(u)\right]_{\mathbf{T}}^{\prime \prime}$ is a monomial in $y_{\left(n, i^{\prime}\right)}\left(i^{\prime}=2, \ldots, n+1\right)$. One can independently study $\left[y_{\mathbf{i}}(u)\right]_{\mathbf{T}}^{\prime}$ and $\left[y_{\mathbf{i}}(u)\right]_{\mathbf{T}}^{\prime \prime}$. The claim (i) follows from the following results, which are proved inductively on $u$ by Lemma 4.1 and the results of $[9,10]$.

(a) $\left[y_{\mathbf{i}}(u)\right]_{\mathbf{T}}^{\prime}$ part. This part is easier. All the monomials $\left[y_{\mathbf{i}}(u)\right]_{\mathbf{T}}^{\prime}$ which are not 1 for $(\mathbf{i}, u): \mathbf{p}_{+}$ in the region $0 \leq u<4 n-2$ are as follows. We have $\left[y_{(i, 1)}(2 i-2)\right]_{\mathbf{T}}^{\prime}=y_{(i, 1)}(i=1, \ldots, n-1)$, and also

$$
\begin{array}{lc}
{\left[y_{(1,1)}(2 n-2)\right]_{\mathbf{T}}^{\prime}=y_{(1,1)}^{-1},} & {\left[y_{(n, 2)}(2 n-1)\right]_{\mathbf{T}}^{\prime}=y_{(1,1)}^{-1},} \\
{\left[y_{(2,1)}(2 n)\right]_{\mathbf{T}}^{\prime}=y_{(1,1)}^{-1} y_{(2,1)}^{-1},} & {\left[y_{(n, 2)}(2 n+1)\right]_{\mathbf{T}}^{\prime}=y_{(2,1)}^{-1},} \\
{\left[y_{(3,1)}(2 n+2)\right]_{\mathbf{T}}^{\prime}=y_{(2,1)}^{-1} y_{(3,1)}^{-1},} & {\left[y_{(n, 2)}(2 n+3)\right]_{\mathbf{T}}^{\prime}=y_{(3,1)}^{-1},} \\
\quad \vdots & \vdots \\
{\left[y_{(n-1,1)}(4 n-6)\right]_{\mathbf{T}}^{\prime}=y_{(n-2,1)}^{-1} y_{(n-1,1)}^{-1},} & {\left[y_{(n, 2)}(4 n-5)\right]_{\mathbf{T}}^{\prime}=y_{(n-1,1)}^{-1},} \\
{\left[y_{(1,1)}(4 n-4)\right]_{\mathbf{T}}^{\prime}=y_{(n-1,1)}^{-1} .} &
\end{array}
$$


(b) $\left[y_{\mathbf{i}}(u)\right]_{\mathbf{T}}^{\prime \prime}$ part. Below we list all the monomials $\left[y_{\mathbf{i}}(u)\right]_{\mathbf{T}}^{\prime \prime}$ which are not 1 for $(\mathbf{i}, u): \mathbf{p}_{+}$in the region $0 \leq u<4 n-2$. We separate the region $0 \leq u<4 n-2$ into four parts corresponding to the decomposition $4 n-2=(2 n-2)+2+(2 n-4)+2$, where $2 n-2$ and $2 n-4$ are the Coxeter numbers of $D_{n}$ and $D_{n-1}$.

Region I: $0 \leq u<2 n-2$. All the monomials $\left[y_{\left(n, i^{\prime}\right)}(u)\right]_{\mathbf{T}}^{\prime \prime}\left(i^{\prime}=2, \ldots, n+1\right)$ for $\left(\left(\mathbf{n}, \mathbf{i}^{\prime}\right), u\right): \mathbf{p}_{+}$ are identified with the positive roots of $D_{n}$ as in [10, Proposition 10.7]; therefore, they are positive. Here, $D_{n}$ is identified with the subgraph of $X_{n}$ consisting of vertices $2, \ldots, n+1$.

Region II: $u=2 n-2,2 n-1$. We have, for even $n$,

$$
\begin{array}{ll}
{\left[y_{\left(n, i^{\prime}\right)}(2 n-2)\right]_{\mathbf{T}}^{\prime \prime}=y_{\left(n, i^{\prime}\right)}^{-1},} & i^{\prime}=3,5, \ldots, n-1, \\
{\left[y_{\left(n, i^{\prime}\right)}(2 n-1)\right]_{\mathbf{T}}^{\prime \prime}=y_{\left(n, i^{\prime}\right)}^{-1},} & i^{\prime}=2,4, \ldots, n, n+1,
\end{array}
$$

and, for odd $n$,

$$
\begin{array}{ll}
{\left[y_{\left(n, i^{\prime}\right)}(2 n-2)\right]_{\mathbf{T}}^{\prime \prime}=y_{\left(n, i^{\prime}\right)}^{-1},} & i^{\prime}=3,5, \ldots, n-2, \\
{\left[y_{(n, n)}(2 n-2)\right]_{\mathbf{T}}^{\prime \prime}=y_{(n, n+1)}^{-1},} & {\left[y_{(n, n+1)}(2 n-2)\right]_{\mathbf{T}}^{\prime \prime}=y_{(n, n)}^{-1},} \\
{\left[y_{\left(n, i^{\prime}\right)}(2 n-1)\right]_{\mathbf{T}}^{\prime \prime}=y_{\left(n, i^{\prime}\right)}^{-1},} & i^{\prime}=2,4, \ldots, n-1 .
\end{array}
$$

Region III: $2 n \leq u<4 n-4$. All the monomials $\left[y_{\left(n, i^{\prime}\right)}(u)\right]_{\mathbf{T}}^{\prime \prime}\left(i^{\prime}=3, \ldots, n+1\right)$ for $\left(\left(n, i^{\prime}\right), u\right)$ : $\mathbf{p}_{+}$are identified with the positive roots of $D_{n-1}$, therefore, they are positive. Here, $D_{n-1}$ is identified with the subgraph of $X_{n}$ consisting of vertices $3, \ldots, n+1$.

Region IV: $u=4 n-4,4 n-3$. We have, for even $n$,

$$
\begin{array}{ll}
{\left[y_{\left(n, i^{\prime}\right)}(4 n-4)\right]_{\mathbf{T}}^{\prime \prime}=y_{\left(n, i^{\prime}\right)}^{-1},} & i^{\prime}=3,5, \ldots, n-1, \\
{\left[y_{\left(n, i^{\prime}\right)}(4 n-3)\right]_{\mathbf{T}}^{\prime \prime}=y_{\left(n, i^{\prime}\right)}^{-1},} & i^{\prime}=2,4, \ldots, n-2, \\
{\left[y_{(n, n)}(4 n-3)\right]_{\mathbf{T}}^{\prime \prime}=y_{(n, n+1)}^{-1},} & {\left[y_{(n, n+1)}(4 n-3)\right]_{\mathbf{T}}^{\prime \prime}=y_{(n, n)}^{-1},}
\end{array}
$$

and, for odd $n$,

$$
\begin{aligned}
& {\left[y_{\left(n, i^{\prime}\right)}(4 n-4)\right]_{\mathbf{T}}^{\prime \prime}=y_{\left(n, i^{\prime}\right)}^{-1}, \quad i^{\prime}=3,5, \ldots, n-2,} \\
& {\left[y_{(n, n)}(4 n-4)\right]_{\mathbf{T}}^{\prime \prime}=y_{(n, n+1)}^{-1}, \quad\left[y_{(n, n+1)}(4 n-4)\right]_{\mathbf{T}}^{\prime \prime}=y_{(n, n)}^{-1},} \\
& {\left[y_{\left(n, i^{\prime}\right)}(4 n-3)\right]_{\mathbf{T}}^{\prime \prime}=y_{\left(n, i^{\prime}\right)}^{-1}, \quad i^{\prime}=2,4, \ldots, n-1 .}
\end{aligned}
$$

Besides, we have the sequences of monomials which appear over Regions III and IV; for even $n$,

$$
\begin{aligned}
& {\left[y_{(2,1)}(2 n)\right]_{\mathbf{T}}^{\prime \prime}=y_{(n, 2)}^{-1} y_{(n, 3)}^{-1}, \quad\left[y_{(3,1)}(2 n+2)\right]_{\mathbf{T}}^{\prime \prime}=y_{(n, 4)}^{-1} y_{(n, 5)}^{-1}, \quad \ldots,} \\
& {\left[y_{(n / 2,1)}(3 n-4)\right]_{\mathbf{T}}^{\prime \prime}=y_{(n, n-2)}^{-1} y_{(n, n-1)}^{-1}, \quad\left[y_{(n / 2+1,1)}(3 n-2)\right]_{\mathbf{T}}^{\prime \prime}=y_{(n, n-1)}^{-1} y_{(n, n)}^{-1} y_{(n, n+1)}^{-1},} \\
& {\left[y_{(n / 2+2,1)}(3 n)\right]_{\mathbf{T}}^{\prime \prime}=y_{(n, n-3)}^{-1} y_{(n, n-2)}^{-1}, \quad \ldots,} \\
& {\left[y_{(n-1,1)}(4 n-6)\right]_{\mathbf{T}}^{\prime \prime}=y_{(n, 3)}^{-1} y_{(n, 4)}^{-1}, \quad\left[y_{(1,1)}(4 n-4)\right]_{\mathbf{T}}^{\prime \prime}=y_{(n, 2)}^{-1},}
\end{aligned}
$$

and, for odd $n$, the middle three terms are replaced with

$$
\begin{aligned}
& {\left[y_{((n-1) / 2,1)}(3 n-5)\right]_{\mathbf{T}}^{\prime \prime}=y_{(n, n-3)}^{-1} y_{(n, n-2)}^{-1},} \\
& {\left[y_{((n+1) / 2,1)}(3 n-3)\right]_{\mathbf{T}}^{\prime \prime}=y_{(n, n-1)}^{-1} y_{(n, n)}^{-1} y_{(n, n+1)}^{-1},} \\
& {\left[y_{(n+3) / 2,1)}(3 n-1)\right]_{\mathbf{T}}^{\prime \prime}=y_{(n, n-2)}^{-1} y_{(n, n-1)}^{-1} .}
\end{aligned}
$$

(ii) They follow from (4.2)-(4.5).

(iii) By $(i)$, for each $\mathbf{i}$ the numbers of the negative monomials $\left[y_{\mathbf{i}}(u)\right]_{\mathrm{T}}$ in the region is 2 for $\mathbf{i}=(1,1), 1$ for $\mathbf{i}=(i, 1)(i=2, \ldots, n-1), n$ for $\mathbf{i}=(n, 2)$. and 2 for $\mathbf{i}=\left(n, i^{\prime}\right)\left(i^{\prime}=3, \ldots, n+1\right)$. Summing up, we have $N_{-}=4 n-2$. 
Now we prove Theorems 2.3 and 2.7. It follows from a very general theorem [13, Theorem 5.1] (based on the work by Plamondon $[29,28]$ ) that the cluster variables $x_{\mathbf{i}}(u)$ and coefficients $y_{\mathbf{i}}(u)$ have the same periodicity with $\left[y_{\mathbf{i}}(u)\right]_{\mathbf{T}}$ as in Proposition $4.2(i i)$, namely, $x_{\mathbf{i}}(4 n-2)=x_{\tau^{-1}(\mathbf{i})}$ and $y_{\mathbf{i}}(4 n-2)=y_{\tau^{-1}(\mathbf{i})}$. It follows that, under the labelling introduced in Lemma 3.3, we have

$$
\begin{array}{ll}
\tilde{x}_{i}(u+4 n-2)=\tilde{x}_{\omega(i)}(u), & (i, u): \mathbf{P}_{+}, \\
y_{i}(u+4 n-2)=y_{\omega(i)}(u), & (i, u): \mathbf{P}_{+}^{\prime},
\end{array}
$$

where $\omega$ is the one in Theorem 2.3 $(i)$. Then, thanks to the isomorphisms in Theorems 3.7 and 3.11, and also by the isomorphisms $\mathcal{T}_{n}^{\circ}(\mathrm{SG})_{+} \simeq \mathcal{T}_{n}^{\circ}(\mathrm{SG})_{-}$and $\mathfrak{y}_{n}^{\circ}(\mathrm{SG})_{+} \simeq y_{n}^{\circ}(\mathrm{SG})_{-}$, we obtain Theorems 2.3 and 2.7 .

Since the F-polynomials (Appendix A(vii)) are defined as a certain specialization of cluster variables, they satisfy the same periodicity as the cluster variables. Let $\tilde{F}_{i}(u)\left((i, u): \mathbf{P}_{+}\right)$be the $F$-polynomial for $\tilde{x}_{i}(u)$. Then, from (4.6) we have

$$
\tilde{F}_{i}(u+4 n-2)=\tilde{F}_{\omega(i)}(u), \quad(i, u): \mathbf{P}_{+} .
$$

Next we prove Theorem 2.4. Let $\bigwedge^{2} \mathbb{P}_{\text {univ }}(y)$ be the quotient of the additive Abelian group $\mathbb{P}_{\text {univ }}(y) \otimes_{\mathbb{Z}} \mathbb{P}_{\text {univ }}(y)$ by the subgroup generated by symmetric tensors $[11,5]$.

Lemma 4.3. In $\bigwedge^{2} \mathbb{P}_{\text {univ }}(y)$, we have

$$
\sum_{\substack{(i, u): \mathbf{P}_{+}^{\prime} \\ 0 \leq u<4 n-2}} y_{i}(u) \wedge\left(1+y_{i}(u)\right)=0 .
$$

Proof. One can prove it in a similar way as in [26, 13], using [10, Proposition 3.13] and (4.7).

Applying the method of $[11,5,26]$, we immediately obtain the following theorem from Lemma 4.3 and Proposition 4.2(iii).

Theorem 4.4. For any semifield homomorphism $\varphi: \mathbb{P}_{\text {univ }}(y) \rightarrow \mathbb{R}_{+}$, we have the following identity.

$$
\frac{6}{\pi^{2}} \sum_{\substack{(i, u): \mathbf{P}_{+}^{\prime} \\ 0 \leq u<4 n-2}} L\left(\frac{\varphi\left(y_{i}(u)\right)}{1+\varphi\left(y_{i}(u)\right)}\right)=4 n-2 .
$$

This is equivalent to Theorem 2.4.

\section{Cluster algebras for RSG Y-systems}

In this section we identify $\mathbb{T}_{n}(\mathrm{RSG})$ and $\mathbb{Y}_{n}(\mathrm{RSG})$ as relations for cluster variables and coefficients of the cluster algebra associated with a certain quiver $Q_{n}(\mathrm{RSG})$. For those things which are quite parallel to the SG case, we skip their precise descriptions just by saying 'as before' unless they are not obvious.

\subsection{Parity decompositions of $\mathrm{T}$ and $\mathrm{Y}$-systems}

For $(i, u) \in \tilde{\mathcal{I}}_{n}$, we set the parity condition $\mathbf{P}_{+}$by, for even $n$,

$$
\mathbf{P}_{+}: i+u \text { is even, } i=1, \ldots, n-2,
$$



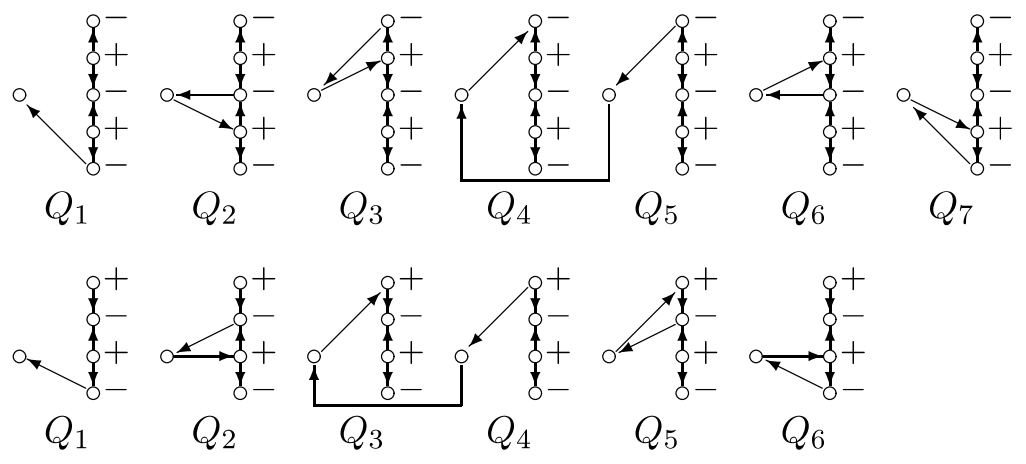

Figure 4. The quiver $Q_{n}(\mathrm{RSG})$ for $n=8$ (upper) and for $n=7$ (lower), where, except for the leftmost vertex of each quiver $Q_{i}$, all the vertices in the same position in $n-1$ quivers $Q_{1}, \ldots, Q_{n-1}$ are identified. We have an arrow between the leftmost vertices of $Q_{4}$ and $Q_{5}$ for $n=8$, and $Q_{3}$ and $Q_{4}$ for $n=7$.

and, for odd $n$,

$$
\mathbf{P}_{+}: \begin{cases}u \text { is even, } & i=1, \\ i+u \text { is even, }, & i=2, \ldots, n-2 .\end{cases}
$$

Define $\tilde{\mathcal{I}}_{n \varepsilon}$ and $\mathcal{T}_{n}^{\circ}(\mathrm{RSG})_{\varepsilon}(\varepsilon= \pm)$ as before. Then, we have $\mathcal{T}_{n}^{\circ}(\mathrm{RSG})_{+} \simeq \mathcal{T}_{n}^{\circ}(\mathrm{RSG})_{-}$by $T_{i}(u) \mapsto T_{i}(u+1)$ and

$$
\mathcal{T}_{n}^{\circ}(\mathrm{RSG}) \simeq \mathcal{T}_{n}^{\circ}(\mathrm{RSG})_{+} \otimes_{\mathbb{Z}} \mathcal{T}_{n}^{\circ}(\mathrm{RSG})_{-} .
$$

For $(i, u) \in \tilde{\mathcal{I}}_{n}$, we set the parity condition $\mathbf{P}_{+}^{\prime}$ by

$$
\mathbf{P}_{+}^{\prime}: i+u \text { is odd, } \quad i=1, \ldots, n-2 .
$$

We have

$$
(i, u): \mathbf{P}_{+}^{\prime} \Longleftrightarrow\left(i, u \pm d_{i}\right): \mathbf{P}_{+},
$$

where $d_{i}$ is given in (2.7). Define $\tilde{\mathcal{I}}_{n \varepsilon}^{\prime}$ and $y_{n}^{\circ}(\mathrm{RSG})_{\varepsilon}(\varepsilon= \pm)$ as before. Then, we have $y_{n}^{\circ}(\mathrm{RSG})_{+} \simeq \mathrm{y}_{n}^{\circ}(\mathrm{RSG})_{-}$by $Y_{i}(u) \mapsto Y_{i}(u+1), 1+Y_{i}(u) \mapsto 1+Y_{i}(u+1)$, and

$$
y_{n}^{\circ}(\mathrm{RSG}) \simeq y_{n}^{\circ}(\mathrm{RSG})_{+} \times y_{n}^{\circ}(\mathrm{RSG})_{-} .
$$

From now on, we mainly treat the + parts, $\mathcal{T}_{n}^{\circ}(\mathrm{RSG})_{+}$and $y_{n}^{\circ}(\mathrm{RSG})_{+} \cdot$

\subsection{Quiver $Q_{n}(\mathrm{RSG})$}

With each $n \geq 4$ we associate a quiver $Q_{n}(\mathrm{RSG})$ as below. First, the cases $n=8$ and $n=7$ are given in Fig. 4, where, except for the leftmost vertex of each quiver $Q_{i}$, all the vertices in the same position in $n-1$ quivers $Q_{1}, \ldots, Q_{n-1}$ are identified. For a general $n$, the quiver $Q_{n}(\mathrm{RSG})$ is defined by naturally extending these examples; in particular, we have an arrow from the leftmost vertex of $Q_{n / 2+1}$ to the leftmost vertex of $Q_{n / 2}$ for even $n$, and an arrow from the leftmost vertex of $Q_{(n+1) / 2}$ to the leftmost vertex of $Q_{(n-1) / 2}$ for odd $n$. Also we assign the property $+/-$ to each vertex, except for the leftmost one in each $Q_{i}$, as in Fig. 4.

Let us choose the index set $\tilde{\mathbf{I}}$ of the vertices of $Q_{n}(\mathrm{RSG})$ naturally obtained from the index set $\mathbf{I}$ of the vertices of $Q_{n}(\mathrm{SG})$ by the restriction to the vertices of $Q_{n}(\mathrm{RSG})$. Thus, $i=1, \ldots, n$; and $i^{\prime}=1$ if $i \neq n$ and $i^{\prime}=2, \ldots, n-2$ if $i=n$. Then, we define $\tilde{\mathbf{I}}_{+}, \tilde{\mathbf{I}}_{-}, \mu_{+}, \mu_{-}$, and $\tilde{w}\left(Q_{n}(\mathrm{RSG})\right)$ as before. 


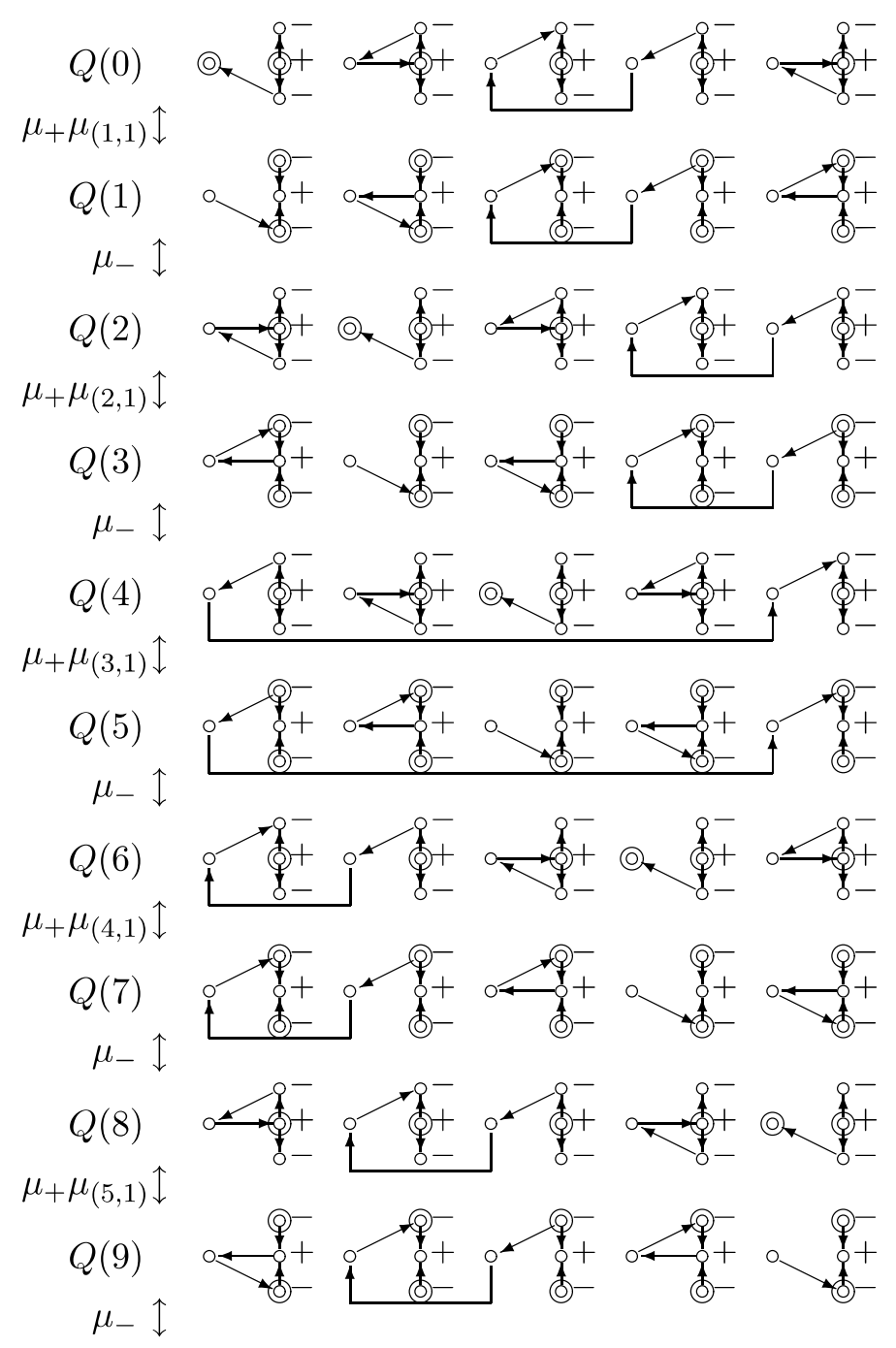

Figure 5. The mutation sequence of the quiver $Q_{n}(\mathrm{RSG})$ in (3.1) for $n=6$. The encircled vertices correspond to the mutation points $(\mathbf{i}, u): \mathbf{p}_{+}$in the forward direction.

Lemma 5.1. Let $Q(0):=Q_{n}(\mathrm{RSG})$. We have the periodic sequence of mutations of quivers (3.1), where the quiver $Q(2 p)(p=1, \ldots, n-2)$ is given by (3.2).

Example 5.2. The mutation sequence (3.1) for $n=6$ is explicitly given in Fig. 5. Let us explain why we need the 'extra arrow' from $Q_{4}$ to $Q_{3}$ in $Q(0)$ in this example. Suppose that we do not have it. Then, we will have an extra arrow from $Q_{3}$ to $Q_{4}$ in $Q(2)$ due to the mutation at $u=1$. This extra arrow will still remain in $Q(4)$, where the vertex $(3,1)$ will be mutated. This conflicts with $\mathbb{T}_{n}(\mathrm{RSG})$ and $\mathbb{Y}_{n}(\mathrm{RSG})$ eventually. The extra arrow is, thus, necessary as a precaution to avoid this confliction.

\section{$5.3 \quad$ Embedding maps}

Let $B=B_{n}(\mathrm{RSG})$ be the skew-symmetric matrix corresponding to the quiver $Q_{n}(\mathrm{RSG})$. Let $\mathcal{A}(B, x, y)$ be the cluster algebra with coefficients in the universal semifield $\mathbb{P}_{\text {univ }}(y)$.

In view of Lemma 5.1 we set $x(0)=x, y(0)=y$ and define clusters $x(u)=\left(x_{\mathbf{i}}(u)\right)_{\mathbf{i} \in \tilde{\mathbf{I}}}(u \in \mathbb{Z})$ and coefficient tuples $y(u)=\left(y_{\mathbf{i}}(u)\right)_{\mathbf{i} \in \tilde{\mathbf{I}}}(u \in \mathbb{Z})$ by the sequence of mutations (3.3) as before. 
For $(\mathbf{i}, u) \in \tilde{\mathbf{I}} \times \mathbb{Z}$, we set the parity condition $\mathbf{p}_{+}$by the same rule as (3.4), where $\mathbf{I}_{ \pm}$is replaced with $\tilde{\mathbf{I}}_{ \pm}$. We define the condition $\mathbf{p}_{-}$by $(\mathbf{i}, u): \mathbf{p}_{-} \Longleftrightarrow(\mathbf{i}, u-1): \mathbf{p}_{+}$.

Lemma 5.3. Below $\equiv$ means the equivalence modulo $(2 n-2) \mathbb{Z}$.

(i) The map

$$
\begin{aligned}
g: & \tilde{\mathcal{I}}_{n+} \rightarrow\left\{(\mathbf{i}, u) \in \tilde{\mathbf{I}} \times \mathbb{Z} \mid(\mathbf{i}, u): \mathbf{p}_{+}\right\} \\
& \left(i, u-d_{i}\right) \mapsto \begin{cases}((j+1,1), u), & i=1 ; u \equiv 2 j(j=0,1, \ldots, n-2), \\
((n, i), u), & i=2, \ldots, n-2\end{cases}
\end{aligned}
$$

is a bijection.

(ii) The map

$$
\begin{aligned}
g^{\prime}: & \tilde{\mathcal{I}}_{n+}^{\prime} \rightarrow\left\{(\mathbf{i}, u) \in \tilde{\mathbf{I}} \times \mathbb{Z} \mid(\mathbf{i}, u): \mathbf{p}_{+}\right\} \\
(i, u) & \mapsto \begin{cases}((j+1,1), u), & i=1 ; u \equiv 2 j(j=0,1, \ldots, n-2), \\
((n, i), u), & i=2, \ldots, n-2\end{cases}
\end{aligned}
$$

is a bijection.

Based on Lemma 5.3, we introduce alternative notations $\tilde{x}_{i}\left(u-d_{i}\right):=x_{\mathbf{i}}(u)$ for $\left(i, u-d_{i}\right) \in \tilde{\mathcal{I}}_{n+}$ with $(\mathbf{i}, u)=g\left(\left(i, u-d_{i}\right)\right)$ and $y_{i}(u):=y_{\mathbf{i}}(u)$ for $(i, u) \in \tilde{\mathcal{I}}_{n+}^{\prime}$ with $(\mathbf{i}, u)=g^{\prime}((i, u))$, respectively.

Let $\mathcal{A}(B, x)$ be the cluster algebra with trivial coefficients.

Definition 5.4. The T-subalgebra $\mathcal{A}_{T}(B, x)$ of $\mathcal{A}(B, x)$ associated with the sequence (3.3) is the subring of $\mathcal{A}(B, x)$ generated by $\left[x_{\mathbf{i}}(u)\right]_{\mathbf{1}}((\mathbf{i}, u) \in \tilde{\mathbf{I}} \times \mathbb{Z})$, or equivalently, generated by $\left[\tilde{x}_{i}(u)\right]_{\mathbf{1}}$ $\left((i, u) \in \tilde{\mathcal{I}}_{n+}\right)$.

By the lemma parallel to Lemma 3.4, we have the following embedding.

Theorem 5.5. The ring $\mathcal{T}_{n}^{\circ}(\mathrm{RSG})_{+}$is isomorphic to $\mathcal{A}_{T}(B, x)$ by the correspondence $T_{i}(u) \mapsto$ $\left[\tilde{x}_{i}(u)\right]_{1}$.

Example 5.6. Consider the case $n=6$ in Fig. 5. Consider the mutation at the vertex $(6,4)$ in $Q(1)$, to which the variable $\tilde{x}_{4}(0)$ is attached. The next time $(6,4)$ is mutated is in $Q(3)$, where $\tilde{x}_{4}(2)$ is attached. Meanwhile, the vertices connected to $(6,4)$ in $Q(1)$ are $(3,1),(4,1)$, $(5,1)$ and $(6,3)$, and the variable attached to them are equal to $\tilde{x}_{1}(-1), \tilde{x}_{1}(1), \tilde{x}_{1}(3)$, and $\tilde{x}_{3}(1)$, respectively. Taking account of the directions of the arrows, we have the relation

$$
\tilde{x}_{4}(0) \tilde{x}_{4}(2)=\frac{y_{4}(1)}{1+y_{4}(1)} \tilde{x}_{1}(-1) \tilde{x}_{1}(3)+\frac{1}{1+y_{4}(1)} \tilde{x}_{1}(1) \tilde{x}_{3}(1),
$$

which agree with (2.13). The other cases are the same as in the SG case.

Let $\mathcal{G}(B, y)$ be the coefficient group associated with $\mathcal{A}(B, x, y)$ as before.

Definition 5.7. The $Y$-subgroup $\mathcal{G}_{Y}(B, y)$ of $\mathcal{G}(B, y)$ associated with the sequence (3.3) is the subgroup of $\mathcal{G}(B, y)$ generated by $y_{\mathbf{i}}(u)((\mathbf{i}, u) \in \tilde{\mathbf{I}} \times \mathbb{Z})$ and $1+y_{\mathbf{i}}(u)\left((\mathbf{i}, u): \mathbf{p}_{+}\right.$or $\left.\mathbf{p}_{-}\right)$, or equivalently, generated by $y_{i}(u)$ and $1+y_{i}(u)\left((i, u) \in \tilde{\mathcal{I}}_{n+}^{\prime}\right)$.

By the lemma parallel to Lemma 3.8, we have the following embedding.

Theorem 5.8. The group $y_{n}^{\circ}(\mathrm{RSG})_{+}$is isomorphic to $\mathcal{G}_{Y}(B, y)$ by the correspondence $Y_{i}(u) \mapsto$ $y_{i}(u)$ and $1+Y_{i}(u) \mapsto 1+y_{i}(u)$. 
Example 5.9. Consider the case $n=6$ in Fig. 5. Let us consider the mutation at the vertex $(1,1)$ in $Q(0)$, to which the variable $y_{1}(0)$ is attached. The next time $(1,1)$ is mutated is in $Q(10)$, where $y_{1}(10)$ is attached. Meanwhile, between $u=0$ and $u=10$, the vertices connected to $(1,1)$ in $Q(u)$ and mutated are $(6,2)$ at $u=1,9,(6,3)$ at $u=2,8$, and $(6,4)$ at $u=3,5,7$. Taking account of the directions of the arrows, we have the relation

$$
y_{1}(0) y_{1}(10)=\frac{\left(1+y_{2}(1)\right)\left(1+y_{2}(9)\right)\left(1+y_{3}(2)\right)\left(1+y_{3}(8)\right)\left(1+y_{4}(3)\right)\left(1+y_{4}(7)\right)}{1+y_{4}(5)^{-1}},
$$

which agrees with (2.10). The other cases are the same as in the SG case.

\section{Proof of Theorems 2.11, 2.12, and 2.15}

In this section we prove Theorems 2.11, 2.12, and 2.15 by the same method as in Section 4 .

Let $B_{n}(\mathrm{RSG})$ be the one in the previous section. The following properties of the tropical Y-system are crucial.

Proposition 6.1. For $\left[\mathcal{G}_{Y}(B, y)\right]_{\mathbf{T}}$ with $B=B_{n}(\mathrm{RSG})$, the following facts hold.

(i) For $0 \leq u<4 n-2$, the monomial $\left[y_{\mathbf{i}}(u)\right]_{\mathbf{T}}\left((\mathbf{i}, u): \mathbf{p}_{+}\right)$is negative if and only if $u$ takes the following values.

$$
\begin{cases}2 n-2 \leq u<4 n-2 & \text { for } \mathbf{i}=(1,1), \ldots,(n-1,1) \\ u=n-2, n-1 ; 2 n-2 \leq u<4 n-2 & \text { for } \mathbf{i}=(n, 2), \\ u=n-2, n-1,2 n-2,2 n-1, & \\ \quad 3 n-3,3 n-2,4 n-4,4 n-3 & \text { for } \mathbf{i}=(n, 3), \ldots,(n, n-2) .\end{cases}
$$

(Note that for each $\mathbf{i}, u$ takes only a part of the list due to the condition $(\mathbf{i}, u): \mathbf{p}_{+} \cdot$ )

(ii) We have $\left[y_{\mathbf{i}}(4 n-2)\right]_{\mathbf{T}}=y_{\tau^{-1}(\mathbf{i})}$, where $\tau$ is a bijection $\mathbf{I} \rightarrow \mathbf{I}$ defined by

$$
\begin{aligned}
& (i, 1) \mapsto(\sigma(i), 1), \quad i=1, \ldots, n-1, \\
& \left(n, i^{\prime}\right) \mapsto\left(n, i^{\prime}\right), \quad i^{\prime}=2, \ldots, n-2,
\end{aligned}
$$

and $\sigma$ is the permutation in (3.2).

(iii) The number $N_{-}$of the negative monomials $\left[y_{\mathbf{i}}(u)\right]_{\mathbf{T}}$ for $(\mathbf{i}, u): \mathbf{p}_{+}$in the region $0 \leq u<$ $4 n-2$ is $6 n-15$.

Proof. (i) Let us factorize $\left[y_{\mathbf{i}}(u)\right]_{\mathbf{T}}=\left[y_{\mathbf{i}}(u)\right]_{\mathbf{T}}^{\prime}\left[y_{\mathbf{i}}(u)\right]_{\mathbf{T}}^{\prime \prime}$, where $\left[y_{\mathbf{i}}(u)\right]_{\mathbf{T}}^{\prime}$ is a monomial in $y_{(i, 1)}$ $(i=1, \ldots, n-1)$ while $\left[y_{\mathbf{i}}(u)\right]_{\mathbf{T}}^{\prime \prime}$ is a monomial in $y_{\left(n, i^{\prime}\right)}\left(i^{\prime}=2, \ldots, n-2\right)$. The claim $(i)$ follows from the following results.

(a) $\left[y_{\mathbf{i}}(u)\right]_{\mathbf{T}}^{\prime}$ part. This part is exactly the same as (4.1) in the $B_{n}(\mathrm{SG})$ case.

(b) $\left[y_{\mathbf{i}}(u)\right]_{\mathbf{T}}^{\prime \prime}$ part. Below we list all the monomials $\left[y_{\mathbf{i}}(u)\right]_{\mathbf{T}}^{\prime \prime}$ which are not 1 for $(\mathbf{i}, u): \mathbf{p}_{+}$in the region $0 \leq u<4 n-2$. We separate the region $0 \leq u<4 n-2$ into eight parts corresponding to the decomposition $4 n-2=(n-2)+2+(n-2)+2+(n-3)+2+(n-3)+2$, where $n-2$ and $n-3$ are the Coxeter numbers of $A_{n-3}$ and $A_{n-4}$.

Region I: $0 \leq u<n-2$. All the monomials $\left[y_{\left(n, i^{\prime}\right)}(u)\right]_{\mathbf{T}}^{\prime \prime}\left(i^{\prime}=2, \ldots, n-2\right)$ for $\left(\left(n, i^{\prime}\right), u\right): \mathbf{p}_{+}$ are identified with the positive roots of $A_{n-3}$; therefore, they are positive. Here, $A_{n-3}$ is identified with the subgraph of $X_{n}$ consisting of vertices $2, \ldots, n-2$.

Region II: $u=n-2, n-1$. We have, for even $n$,

$$
\left[y_{\left(n, i^{\prime}\right)}(n-2)\right]_{\mathbf{T}}^{\prime \prime}=y_{\left(n, n-i^{\prime}\right)}^{-1}, \quad i^{\prime}=3,5, \ldots, n-3,
$$




$$
\left[y_{\left(n, i^{\prime}\right)}(n-1)\right]_{\mathbf{T}}^{\prime \prime}=y_{\left(n, n-i^{\prime}\right)}^{-1}, \quad i^{\prime}=2,4, \ldots, n-2,
$$

and, for odd $n$,

$$
\begin{array}{ll}
{\left[y_{\left(n, i^{\prime}\right)}(n-2)\right]_{\mathbf{T}}^{\prime \prime}=y_{\left(n, n-i^{\prime}\right)}^{-1},} & i^{\prime}=2,4, \ldots, n-3, \\
{\left[y_{\left(n, i^{\prime}\right)}(n-1)\right]_{\mathbf{T}}^{\prime \prime}=y_{\left(n, n-i^{\prime}\right)}^{-1},} & i^{\prime}=3,5, \ldots, n-2 .
\end{array}
$$

Region III: $n \leq u<2 n-2$. Once again, all the monomials $\left[y_{\left(n, i^{\prime}\right)}(u)\right]_{\mathbf{T}}^{\prime \prime}\left(i^{\prime}=2, \ldots, n-2\right)$ for $\left(\left(n, i^{\prime}\right), u\right): \mathbf{p}_{+}$are identified with the positive roots of $A_{n-3}$; therefore, they are positive.

Region IV: $n=2 n-2,2 n-1$. We have

$$
\begin{array}{ll}
{\left[y_{\left(n, i^{\prime}\right)}(2 n-2)\right]_{\mathbf{T}}^{\prime \prime}=y_{\left(n, i^{\prime}\right)}^{-1},} & i^{\prime}=3,5, \ldots, \\
{\left[y_{\left(n, i^{\prime}\right)}(2 n-1)\right]_{\mathbf{T}}^{\prime \prime}=y_{\left(n, i^{\prime}\right)}^{-1},} & i^{\prime}=2,4, \ldots .
\end{array}
$$

Region V: $2 n \leq u<3 n-3$. All the monomials $\left[y_{\left(n, i^{\prime}\right)}(u)\right]_{\mathbf{T}}^{\prime \prime}\left(i^{\prime}=3, \ldots, n-2\right)$ for $\left(\left(n, i^{\prime}\right), u\right)$ : $\mathbf{p}_{+}$are identified with the positive roots of $A_{n-4}$; therefore, they are positive. Here, $A_{n-4}$ is identified with the subgraph of $X_{n}$ consisting of vertices $3, \ldots, n-2$.

Region VI: $u=3 n-3,3 n-2$. We have, for even $n$,

$$
\begin{array}{ll}
{\left[y_{\left(n, i^{\prime}\right)}(3 n-3)\right]_{\mathbf{T}}^{\prime \prime}=y_{\left(n, n+1-i^{\prime}\right)}^{-1},} & i^{\prime}=4,6, \ldots, n-2, \\
{\left[y_{\left(n, i^{\prime}\right)}(3 n-2)\right]_{\mathbf{T}}^{\prime \prime}=y_{\left(n, n+1-i^{\prime}\right)}^{-1},} & i^{\prime}=3,5, \ldots, n-3,
\end{array}
$$

and, for odd $n$,

$$
\begin{array}{ll}
{\left[y_{\left(n, i^{\prime}\right)}(3 n-3)\right]_{\mathbf{T}}^{\prime \prime}=y_{\left(n, n+1-i^{\prime}\right)}^{-1},} & i^{\prime}=3,5, \ldots, n-2, \\
{\left[y_{\left(n, i^{\prime}\right)}(3 n-2)\right]_{\mathbf{T}}^{\prime \prime}=y_{\left(n, n+1-i^{\prime}\right)}^{-1},} & i^{\prime}=4,6, \ldots, n-3 .
\end{array}
$$

Region VII: $3 n-1 \leq u<4 n-4$. Once again, all the monomials $\left[y_{\left(n, i^{\prime}\right)}(u)\right]_{\mathbf{T}}^{\prime \prime}\left(i^{\prime}=3, \ldots, n-2\right)$ for $\left(\left(n, i^{\prime}\right), u\right): \mathbf{p}_{+}$are identified with the positive roots of $A_{n-4}$; therefore, they are positive.

Region VIII: $u=4 n-4,4 n-3$. We have

$$
\begin{array}{ll}
{\left[y_{\left(n, i^{\prime}\right)}(4 n-4)\right]_{\mathbf{T}}^{\prime \prime}=y_{\left(n, i^{\prime}\right)}^{-1},} & i^{\prime}=3,5, \ldots, \\
{\left[y_{\left(n, i^{\prime}\right)}(4 n-3)\right]_{\mathbf{T}}^{\prime \prime}=y_{\left(n, i^{\prime}\right)}^{-1},} & i^{\prime}=2,4, \ldots .
\end{array}
$$

Besides, we have the sequences of monomials which appear over Regions V-VIII; for even $n$,

$$
\begin{aligned}
& \left.\left[y_{(2,1)}(2 n)\right]_{\mathbf{T}}^{\prime \prime}=y_{(n, 2)}^{-1} y_{(n, 3)}^{-1}, \quad y_{(3,1)}(2 n+2)\right]_{\mathbf{T}}^{\prime \prime}=y_{(n, 4)}^{-1} y_{(n, 5)}^{-1}, \quad \ldots, \\
& {\left[y_{(n / 2,1)}(3 n-4)\right]_{\mathbf{T}}^{\prime \prime}=y_{(n, n-2)}^{-1}, \quad\left[y_{(n / 2+2,1)}(3 n)\right]_{\mathbf{T}}^{\prime \prime}=y_{(n, n-3)}^{-1} y_{(n, n-2)}^{-1}, \quad \ldots,} \\
& {\left[y_{(n-1,1)}(4 n-6)\right]_{\mathbf{T}}^{\prime \prime}=y_{(n, 3)}^{-1} y_{(n, 4)}^{-1}, \quad\left[y_{(1,1)}(4 n-4)\right]_{\mathbf{T}}^{\prime \prime}=y_{(n, 2)}^{-1},}
\end{aligned}
$$

and, for odd $n$, the middle two terms are replaced with

$$
\begin{aligned}
& {\left[y_{((n-1) / 2,1)}(3 n-5)\right]_{\mathbf{T}}^{\prime \prime}=y_{(n, n-3)}^{-1} y_{(n, n-2)}^{-1},} \\
& {\left[y_{(n+3) / 2,1)}(3 n-1)\right]_{\mathbf{T}}^{\prime \prime}=y_{(n, n-2)}^{-1} .}
\end{aligned}
$$

(ii) They follow from (6.1)-(6.3).

(iii) By $(i)$, for each $\mathbf{i}$ the numbers of the negative monomials $\left[y_{\mathbf{i}}(u)\right]_{\mathrm{T}}$ in the region is 2 for $\mathbf{i}=(1,1), 1$ for $\mathbf{i}=(i, 1)(i=2, \ldots, n-1), n+1$ for $\mathbf{i}=(n, 2)$. and 4 for $\mathbf{i}=\left(n, i^{\prime}\right)$ $\left(i^{\prime}=3, \ldots, n-2\right)$. Summing up, we have $N_{-}=6 n-15$.

Theorems 2.11, 2.15 and 2.12 follow from Proposition 6.1 as before. 


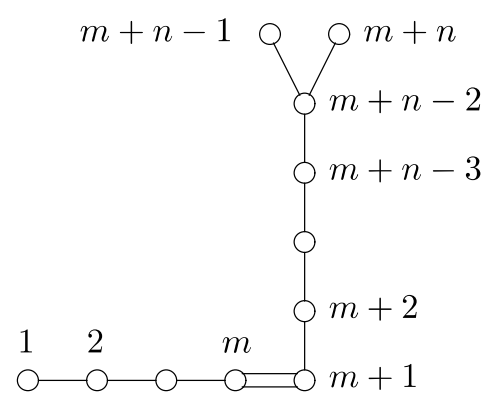

Figure 6. The diagram $X_{m, n}$.

\section{$7 \quad$ Further extension}

Seeing that the cluster algebraic setting perfectly works for the case (2.2), it is rather natural to expect that the method in this paper is, at least in principle, applicable for a general rational $\xi$. On the other hand, working out in full generality seems a complicated task, and we do not pursue it in this paper. However, repeating our method to a little more general case

$$
\xi=\frac{n-1}{m n-m+1}=\frac{1}{m+\frac{1}{n-1}}
$$

is not difficult. Since the proof is mostly the repetition of the previous case $m=1$, we concentrate on exhibiting the results.

\subsection{Further result for SG Y-systems}

With a pair of integers $m \geq 1$ and $n \geq 4$, we associate a diagram $X_{m, n}$ in Fig. 6, following [32]. Let $\mathcal{I}_{m, n}=\{1, \ldots, m+n\} \times \mathbb{Z}$.

Definition 7.1 ([32]). Fix a pair of integers $m \geq 1$ and $n \geq 4$. The sine-Gordon (SG) Y-system $\mathbb{Y}_{m, n}(\mathrm{SG})$ is the following system of relations for a family of variables $\left\{Y_{i}(u) \mid(i, u) \in \mathcal{I}_{m, n}\right\}$,

$$
\begin{aligned}
& Y_{i}(u-n+1) Y_{i}(u+n-1)=\prod_{j: j \sim i}\left(1+Y_{j}(u)\right), \quad i=1, \ldots, m-1, \\
& Y_{m}(u-n+1) Y_{m}(u+n-1)=\left(1+Y_{m-1}(u)\right) \\
& \quad \times\left(\prod_{j=m+1}^{m+n-2}\left(1+Y_{j}(u-m-n+1+j)\right)\left(1+Y_{j}(u+m+n-1-j)\right)\right) \\
& \quad \times\left(1+Y_{m+n-1}(u)\right)\left(1+Y_{m+n}(u)\right), \\
& Y_{m+1}(u-1) Y_{m+1}(u+1)=\frac{1+Y_{m}(u)}{1+Y_{m+2}(u)^{-1}}, \\
& Y_{i}(u-1) Y_{i}(u+1)=\frac{1}{\prod_{j: j \sim i}\left(1+Y_{j}(u)^{-1}\right)}, \quad i=m+2, \ldots, m+n,
\end{aligned}
$$

where $j \sim i$ means that $j$ is adjacent to $i$ in $X_{m, n}$.

Definition 7.2. Fix a pair of integers $m \geq 1$ and $n \geq 4$. The sine-Gordon (SG) T-system $\mathbb{T}_{m, n}(\mathrm{SG})$ is the following system of relations for a family of variables $\left\{T_{i}(u) \mid(i, u) \in \mathcal{I}_{m, n}\right\}$,

$$
T_{i}(u-n+1) T_{i}(u+n-1)=\prod_{j: j \sim i} T_{j}(u)+1, \quad i=1, \ldots, m,
$$




$$
\begin{aligned}
& T_{m+1}(u-1) T_{m+1}(u+1)=T_{m}(u-n+2) T_{m}(u+n-2)+T_{m+2}(u), \\
& \begin{aligned}
T_{i}(u-1) T_{i}(u+1)= & T_{m}(u-m-n+1+i) \\
& \times T_{m}(u+m+n-1-i)+\prod_{j: j \sim i} T_{j}(u), \quad i=m+2, \ldots, m+n-2,
\end{aligned} \\
& T_{m+n-1}(u-1) T_{m+n-1}(u+1)=T_{m}(u)+T_{m+n-2}(u), \\
& T_{m+n}(u-1) T_{m+n}(u+1)=T_{m}(u)+T_{m+n-2}(u),
\end{aligned}
$$

where $j \sim i$ means that $j$ is adjacent to $i$ in $X_{m, n}$.

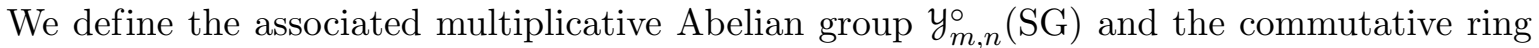
$\mathfrak{T}_{m, n}^{\circ}(\mathrm{SG})$ as before.

\section{Theorem 7.3.}

(i) (Conjectured by [32].) The following relations hold in $y_{m, n}^{\circ}(\mathrm{SG})$.

(a) Suppose that both $m$ and $n$ are even. (In other words, $m n-m+n$ is even.) Then, we have the periodicity: $Y_{i}(u+2(m n-m+n))=Y_{i}(u)$. (There is no half periodicity.)

(b) Suppose that at least one of $m$ or $n$ is odd. (In other words, $m n-m+n$ is odd.) Then, we have the half periodicity: $Y_{i}(u+2(m n-m+n))=Y_{\omega(i)}(u)$, where $\omega$ is an involution of the set $\{1, \ldots, m+n\}$ defined by $\omega(m+n-1)=m+n, \omega(m+n)=$ $m+n-1$, and $\omega(i)=i(i=1, \ldots, m+n-2)$. Therefore, we have the full periodicity: $Y_{i}(u+4(m n-m+n))=Y_{i}(u)$.

(ii) The same periodicity holds in $\mathcal{T}_{m, n}^{\circ}(\mathrm{SG})$ by replacing $Y_{i}(u)$ in $(i)$ with $T_{i}(u)$.

(iii) (Conjectured by [32].) Suppose that a family of positive real numbers $\left\{Y_{i}(u) \mid(i, u) \in \mathcal{I}_{n}\right\}$ satisfies $\mathbb{Y}_{m, n}(\mathrm{SG})$. Then, we have the identities

$$
\begin{aligned}
& \frac{6}{\pi^{2}} \sum_{\substack{(i, u) \in \mathcal{I}_{m, n} \\
0 \leq u<4(m n-m+n)}} L\left(\frac{Y_{i}(u)}{1+Y_{i}(u)}\right)=4(m+1)(m n-m+n), \\
& \frac{6}{\pi^{2}} \sum_{\substack{(i, u) \in \mathcal{I}_{m, n} \\
0 \leq u<4(m n-m+n)}} L\left(\frac{1}{1+Y_{i}(u)}\right)=4(n-1)(m n-m+n) .
\end{aligned}
$$

In our proof of Theorem 7.3 we have a natural interpretation of the full/half period

$$
2(m n-m+n)=h\left(D_{n}\right)+2+m\left(h\left(D_{n-1}\right)+2\right) .
$$

Remark 7.4. Actually, $\mathbb{Y}_{m, n}(\mathrm{SG})$ and $\mathbb{T}_{m, n}(\mathrm{SG})$ are also considered for $n=3$, and they coincide with the $\mathrm{Y}$ and T-systems of type $B_{m+1}$ with level 2 in [24]. Theorem 7.3 remains valid for $n=3$ due to [13].

The cluster algebraic formulation of $\mathbb{Y}_{m, n}(\mathrm{SG})$ and $\mathbb{T}_{m, n}(\mathrm{SG})$ is done by the quiver $Q_{m, n}(\mathrm{SG})$ defined as follows. As a rather general example, the case $(m, n)=(4,7)$ is given in Fig. 7, where, all the vertices with $\bullet$ in the same position in the quivers $Q_{1}, \ldots, Q_{6}$ are identified. Note that the arrows between the vertices with $\circ$ in $Q_{1}, Q_{2}, Q_{3}$ and $Q_{4}, Q_{5}, Q_{6}$ are opposite. For a general $n$, the quiver $Q_{n}(\mathrm{SG})$ is defined by naturally extending this example. Namely, add $m-1$ vertices to the left of the leftmost vertex in each quiver $Q_{i}$ of $Q_{n}(\mathrm{SG})$. We assign the property $+/-$ to each vertex as in Fig. 7 such that the leftmost vertex of each $Q_{i}$ has the property - for even $m$ and + for odd $m$. Then, we put the arrows between the vertices with $\circ$ such that, for even $n$, 

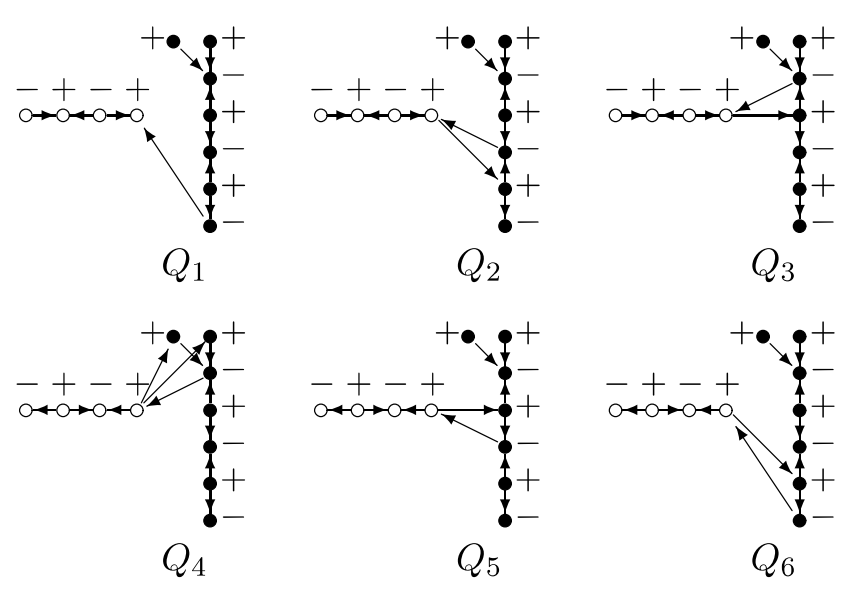

Figure 7. The quiver $Q_{m, n}(\mathrm{SG})$ for $(m, n)=(4,7)$, where, all the vertices with $\bullet$ in the same position in the quivers $Q_{1}, \ldots, Q_{6}$ are identified. Note that the arrows between the vertices with $\circ$ in $Q_{1}, Q_{2}, Q_{3}$ and $Q_{4}, Q_{5}, Q_{6}$ are opposite.

each vertex with property $(\circ,-)$ is a source for $Q_{1}, \ldots, Q_{n / 2}$ and a sink for $Q_{n / 2+1}, \ldots, Q_{n-1}$; and, for odd $n$, each vertex with property $(\circ,-)$ is a source for $Q_{1}, \ldots, Q_{(n-1) / 2}$ and a sink for $Q_{(n+1) / 2+1}, \ldots, Q_{n-1}$.

Let us choose the index set $\mathbf{I}$ of the vertices of $Q_{m, n}(\mathrm{SG})$ so that, under the natural identification with $X_{m, n}, \mathbf{i}=\left(i, i^{\prime}\right) \in \mathbf{I}\left(i=1, \ldots, n-1 ; i^{\prime}=1, \ldots, m\right)$ represents the vertex with $\circ$ in $Q_{i}$, and $\mathbf{i}=\left(n, i^{\prime}\right) \in \mathbf{I}\left(i^{\prime}=m+1, \ldots, m+n\right)$ represents the vertex with $\bullet$ in any quiver $Q_{i}$.

Let $\mathbf{I}_{+}^{\bullet}\left(\right.$ resp. $\left.\mathbf{I}_{-}^{\bullet}\right)$ denote the set of the vertices with property $(\bullet,+)($ resp. $(\bullet,-))$. Let $\mathbf{I}_{+, i}^{\circ}$ $\left(\right.$ resp. $\left.\mathbf{I}_{-, i}^{\circ}\right)$ denote the set of the vertices with property $(\circ,+)($ resp. $(\circ,-))$ in the $i$ th quiver $Q_{i}$.

We define composite mutations,

$$
\mu_{+}^{\bullet}=\prod_{\mathbf{i} \in \mathbf{I}_{+}^{\bullet}} \mu_{\mathbf{i}}, \quad \mu_{-}^{\bullet}=\prod_{\mathbf{i} \in \mathbf{I}_{-}^{\bullet}} \mu_{\mathbf{i}}, \quad \mu_{+, i}^{\circ}=\prod_{\mathbf{i} \in \mathbf{I}_{+, i}^{\circ}} \mu_{\mathbf{i}}, \quad \mu_{-, i}^{\circ}=\prod_{\mathbf{i} \in \mathbf{I}_{-, i}^{\circ}} \mu_{\mathbf{i}} .
$$

Lemma 7.5. Let $Q(0):=Q_{m, n}(\mathrm{SG})$. We have the following periodic sequence of mutations of quivers: for even $n$,

$$
\begin{aligned}
Q(0) & \stackrel{\mu_{+}^{\bullet} \mu_{+, 1}^{\circ}}{\longleftrightarrow} Q(1) \stackrel{\mu_{-}^{\bullet} \mu_{-, n / 2+1}^{\circ}}{\longleftrightarrow} Q(2) \stackrel{\mu_{+}^{\bullet} \mu_{+, 2}^{\circ}}{\longleftrightarrow} Q(3) \stackrel{\mu_{-}^{\bullet} \mu_{-, n / 2+2}^{\circ}}{\longleftrightarrow} \ldots \\
& \stackrel{\mu_{+}^{\bullet} \mu_{+, n / 2}^{\circ}}{\longleftrightarrow} Q(n-1) \stackrel{\mu_{-}^{\bullet} \mu_{-, 1}^{\circ}}{\longleftrightarrow} Q(n) \stackrel{\mu_{+}^{\bullet} \mu_{+, n / 2+1}^{\circ}}{\longleftrightarrow} Q(n+1) \stackrel{\mu_{-}^{\bullet} \mu_{-, 2}^{\circ}}{\longleftrightarrow} \ldots \\
& \stackrel{\mu_{-}^{\bullet} \mu_{-, n / 2-1}^{\circ}}{\longleftrightarrow} Q(2 n-4) \stackrel{\mu_{+}^{\bullet} \mu_{+, n-1}^{\circ}}{\longleftrightarrow} Q(2 n-3) \stackrel{\mu_{-}^{\bullet} \mu_{-, n / 2}^{\circ}}{\longleftrightarrow} Q(2 n-2)=Q(0),
\end{aligned}
$$

and, for odd $n$,

$$
\begin{aligned}
Q(0) & \stackrel{\mu_{+}^{\bullet} \mu_{+, 1}^{\circ} \mu_{-,(n+1) / 2}^{\circ}}{\longleftrightarrow} Q(1) \stackrel{\mu_{-}^{\bullet}}{\longleftrightarrow} Q(2) \stackrel{\mu_{+}^{\bullet} \mu_{+, 2}^{\circ} \mu_{-,(n+3) / 2}^{\circ}}{\longleftrightarrow} Q(3) \stackrel{\mu_{-}^{\bullet}}{\longleftrightarrow} \ldots \\
& \stackrel{\mu_{-}^{\bullet}}{\longleftrightarrow} Q(n-1) \stackrel{\mu_{+}^{\bullet} \mu_{+,(n+1) / 2}^{\circ} \mu_{-, 1}^{\circ}}{\longleftrightarrow} Q(n) \stackrel{\mu_{-}^{\bullet}}{\longleftrightarrow} Q(n+1) \stackrel{\mu_{+}^{\bullet} \mu_{+,(n+3) / 2}^{\circ} \mu_{-, 2}^{\circ}}{\longleftrightarrow} \ldots \\
& \stackrel{\mu_{-}^{\bullet}}{\longleftrightarrow} Q(2 n-4) \stackrel{\mu_{+}^{\bullet} \mu_{+, n-1}^{\circ} \mu_{-,(n-1) / 2}^{\circ}}{\longleftrightarrow} Q(2 n-3) \stackrel{\mu_{-}^{\bullet}}{\longleftrightarrow} Q(2 n-2)=Q(0) .
\end{aligned}
$$

For $(\mathbf{i}, u) \in \mathbf{I} \times \mathbb{Z}$, write $(\mathbf{i}, u): \mathbf{p}_{+}$if $(\mathbf{i}, u)$ is the forward mutation points in (7.3) for even $n$ and in $(7.4)$ for odd $n$, modulo $(2 n-2) \mathbb{Z}$ for $u$. Then, one can repeat and extend all the arguments for $m=1$ in Sections 3 and 4, prove the following proposition, and obtain Theorem 7.3.

Proposition 7.6. For $\left[\mathcal{G}_{Y}(B, y)\right]_{\mathbf{T}}$ with $B=B_{m, n}(\mathrm{SG})$, the following facts hold. 
(i) For $0 \leq u<2(m n-m+n)$, the monomial $\left[y_{\mathbf{i}}(u)\right]_{\mathbf{T}}\left((\mathbf{i}, u): \mathbf{p}_{+}\right)$is negative if and only if $u$ takes the values $2 n-2 \leq u<2(m n-m+n)$ for $\mathbf{i}=\left(i, i^{\prime}\right)\left(i=1, \ldots, n-1 ; i^{\prime}=\right.$ $1, \ldots, m)$ and for $\mathbf{i}=(n, m+1)$, and $u=2 k(n-1), 2 k(n-1)+1(k=1, \ldots, m+1)$ for $\mathbf{i}=(n, m+2), \ldots,(n, m+n)$.

(ii) We have $\left[y_{\mathbf{i}}(2(m n-m+n))\right]_{\mathbf{T}}=y_{\tau^{-1}(\mathbf{i})}$, where $\tau$ is a bijection $\mathbf{I} \rightarrow \mathbf{I}$ defined as follows. If both $m$ and $n$ are even,

$$
\begin{aligned}
& \left(i, i^{\prime}\right) \mapsto\left(\sigma(i), i^{\prime}\right), \quad i=1, \ldots, n-1, \quad i^{\prime}=1, \ldots, m, \\
& \left(n, i^{\prime}\right) \mapsto\left(n, i^{\prime}\right), \quad i^{\prime}=m+1, \ldots, m+n
\end{aligned}
$$

and $\sigma$ is the permutation in (3.2). If at least one of $m$ or $n$ is odd, we modify $\tau$ in (7.5) by $(n, m+n-1) \mapsto(n, m+n)$ and $(n, m+n) \mapsto(n, m+n-1)$. (The rest are the same as in (7.5).)

(iii) The number $N_{-}$of the negative monomials $\left[y_{\mathbf{i}}(u)\right]_{\mathbf{T}}$ for $(\mathbf{i}, u): \mathbf{p}_{+}$in the region $0 \leq u<$ $2(m n-m+n)$ is $(m+1)(m n-m+n)$.

\subsection{Further result for RSG Y-system}

The RSG case is quite similar. Let $\tilde{\mathcal{I}}_{m, n}=\{1, \ldots, m+n-3\} \times \mathbb{Z}$.

Definition 7.7 ([32]). Fix a pair of integers $m \geq 1$ and $n \geq 4$. The reduced sine-Gordon (RSG) Y-system $\mathbb{Y}_{m, n}(\mathrm{RSG})$ is the following system of relations for a family of variables $\left\{Y_{i}(u) \mid(i, u) \in\right.$ $\left.\tilde{\mathcal{I}}_{m, n}\right\}$,

$$
\begin{aligned}
& Y_{i}(u-n+1) Y_{i}(u+n-1)=\prod_{j: j \sim i}\left(1+Y_{j}(u)\right), \quad i=1, \ldots, m-1, \\
& Y_{m}(u-n+1) Y_{m}(u+n-1)=\left(1+Y_{m-1}(u)\right) \\
& \quad \times\left(\prod_{j=m+1}^{m+n-3}\left(1+Y_{j}(u-m-n+1+j)\right)\left(1+Y_{j}(u+m+n-1-j)\right)\right) \\
& \quad \times\left(1+Y_{m+n-3}(u)^{-1}\right)^{-1}, \\
& Y_{m+1}(u-1) Y_{m+1}(u+1)=\frac{1+Y_{m}(u)}{1+Y_{m+2}(u)^{-1}}, \\
& Y_{i}(u-1) Y_{i}(u+1)=\frac{1}{\prod_{j: j \sim i}\left(1+Y_{j}(u)^{-1}\right)}, \quad i=m+2, \ldots, m+n-3,
\end{aligned}
$$

where $j \sim i$ means that $j \leq m+n-3$ is adjacent to $i$ in $X_{m, n}$.

Definition 7.8. Fix a pair of integers $m \geq 1$ and $n \geq 4$. The reduced sine-Gordon (RSG) Tsystem $\mathbb{T}_{m, n}(\mathrm{RSG})$ is the following system of relations for a family of variables $\left\{T_{i}(u) \mid(i, u) \in\right.$ $\left.\tilde{\mathcal{I}}_{m, n}\right\}$,

$$
\begin{aligned}
& T_{i}(u-n+1) T_{i}(u+n-1)=\prod_{j: j \sim i} T_{j}(u)+1, \quad i=1, \ldots, m, \\
& \begin{aligned}
& T_{m+1}(u-1) T_{m+1}(u+1)=T_{m}(u-n+2) T_{m}(u+n-2)+T_{m+2}(u), \\
& T_{i}(u-1) T_{i}(u+1)= T_{m}(u-m-n+1+i) \\
& \times T_{m}(u+m+n-1-i)+\prod_{j: j \sim i} T_{j}(u), \quad i=m+2, \ldots, m+n-4,
\end{aligned} \\
& T_{m+n-3}(u-1) T_{m+n-3}(u+1)=T_{m}(u-2) T_{m}(u+2)+T_{m}(u) T_{m+n-4}(u),
\end{aligned}
$$

where $j \sim i$ means that $j \leq m+n-3$ is adjacent to $i$ in $X_{m, n}$. 

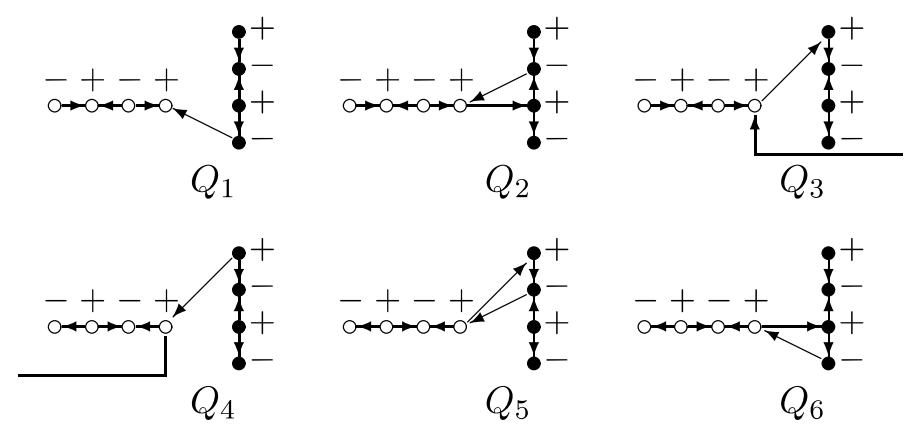

Figure 8. The quiver $Q_{m, n}(\mathrm{RSG})$ for $(m, n)=(4,7)$, where, all the vertices with $\bullet$ in the same position in the quivers $Q_{1}, \ldots, Q_{6}$ are identified. We have an arrow between the fourth vertices (from the left) of $Q_{3}$ and $Q_{4}$.

We define the associated multiplicative Abelian group $\underset{m, n}{\circ}(\mathrm{RSG})$ and the commutative ring $\mathcal{T}_{m, n}^{\circ}(\mathrm{RSG})$ as before.

\section{Theorem 7.9.}

(i) (Conjectured by [32].) The following relations hold in $y_{m, n}^{\circ}(\mathrm{RSG})$.

Periodicity: $Y_{i}(u+2(m n-m+n))=Y_{i}(u)$.

(ii) The following relations hold in $\mathcal{T}_{m, n}^{\circ}(\mathrm{RSG})$.

Periodicity: $T_{i}(u+2(m n-m+n))=T_{i}(u)$.

(iii) (Conjectured by [32].) Suppose that a family of positive real numbers $\left\{Y_{i}(u) \mid(i, u) \in \mathcal{I}_{n}\right\}$ satisfies $\mathbb{Y}_{m, n}(\mathrm{RSG})$. Then, we have the identities

$$
\begin{aligned}
& \frac{6}{\pi^{2}} \sum_{\substack{(i, u) \in \mathcal{I}_{m, n} \\
0 \leq u<2(m n-m+n)}} L\left(\frac{Y_{i}(u)}{1+Y_{i}(u)}\right)=2\left(n m^{2}-m^{2}+3 m n-8 m+2 n-6\right), \\
& \frac{6}{\pi^{2}} \sum_{\substack{(i, u) \in \mathcal{I}_{m, n} \\
0 \leq u<2(m n-m+n)}} L\left(\frac{1}{1+Y_{i}(u)}\right)=2\left(n^{2} m-6 n m+11 m+n^{2}-5 n+6\right) .
\end{aligned}
$$

In our proof of Theorem 7.3 we have a natural interpretation of the period

$$
2(m n-m+n)=2\left\{h\left(A_{n-3}\right)+2+m\left(h\left(A_{n-4}\right)+2\right)\right\} .
$$

The cluster algebraic formulation of $\mathbb{Y}_{m, n}(\mathrm{RSG})$ and $\mathbb{T}_{m, n}(\mathrm{RSG})$ is done by the quiver $Q_{m, n}(\mathrm{RSG})$ defined as follows. Add $m-1$ vertices to the left of the leftmost vertex in each quiver $Q_{i}$ of $Q_{n}(\mathrm{RSG})$ and put the arrows between them exactly in the same way as in the quiver $Q_{m, n}(\mathrm{SG})$. As an example, the case $(m, n)=(4,7)$ is given in Fig. 8. We also assign the properties $+/-$ and $\circ / \bullet$ to each vertex in a similar way to $Q_{m, n}(\mathrm{SG})$ as in Fig. 8.

Let us choose the index set $\tilde{\mathbf{I}}$ of the vertices of $Q_{m, n}(\mathrm{RSG})$ naturally obtained from the index set $\mathbf{I}$ of the vertices of $Q_{m, n}(\mathrm{SG})$ by the restriction to the vertices of $Q_{m, n}(\mathrm{RSG})$. Thus, $\mathbf{i}=\left(i, i^{\prime}\right) \in \tilde{\mathbf{I}}\left(i=1, \ldots, n-1 ; i^{\prime}=1, \ldots, m\right)$ represents the vertex with $\circ$ in $Q_{i}$, and $\mathbf{i}=\left(n, i^{\prime}\right) \in \tilde{\mathbf{I}}$ $\left(i^{\prime}=m+1, \ldots, m+n-3\right)$ represents the vertex with $\bullet$ in any quiver $Q_{i}$. Then, we define $\tilde{\mathbf{I}}_{+}^{\bullet}$, $\tilde{\mathbf{I}}_{-}^{\bullet}, \tilde{\mathbf{I}}_{+, i}^{\circ}, \tilde{\mathbf{I}}_{-, i}^{\circ}$ and the corresponding mutations by (7.2).

Lemma 7.10. Let $Q(0):=Q_{m, n}(\mathrm{RSG})$. We have the periodic sequence of mutations of quivers (7.3) for even $n$ and quivers (7.4) for odd $n$. 
For $(\mathbf{i}, u) \in \mathbf{I} \times \mathbb{Z}$, write $(\mathbf{i}, u): \mathbf{p}_{+}$if $(\mathbf{i}, u)$ is the forward mutation points in (7.3) for even $n$ and in (7.4) for odd $n$, modulo $(2 n-2) \mathbb{Z}$ for $u$. Then, one can repeat and extend all the arguments for $m=1$ in Sections 5 and 6, prove the following proposition, and obtain Theorem 7.9.

Proposition 7.11. For $\left[\mathcal{G}_{Y}(B, y)\right]_{\mathbf{T}}$ with $B=B_{m, n}(\mathrm{RSG})$, the following facts hold.

(i) For $0 \leq u<2(m n-m+n)$, the monomial $\left[y_{\mathbf{i}}(u)\right]_{\mathbf{T}}\left((\mathbf{i}, u): \mathbf{p}_{+}\right)$is negative if and only if $u$ takes the values $2 n-2 \leq u<2(m n-m+n)$ for $\mathbf{i}=\left(i, i^{\prime}\right)\left(i=1, \ldots, n-1 ; i^{\prime}=\right.$ $1, \ldots, m), u=n-2, n-1,2 n-2 \leq u<2(m n-m+n)$ for $\mathbf{i}=(n, m+1)$, and $u=n-2, n-1, k(n-1), k(n-1)+1(k=2, \ldots, 2 m+2)$ for $\mathbf{i}=(n, m+2), \ldots,(n, m+n-3)$.

(ii) We have $\left[y_{\mathbf{i}}(2(m n-m+n))\right]_{\mathbf{T}}=y_{\tau^{-1}(\mathbf{i})}$, where $\tau$ is a bijection $\tilde{\mathbf{I}} \rightarrow \tilde{\mathbf{I}}$ defined by

$$
\begin{aligned}
& \left(i, i^{\prime}\right) \mapsto\left(\sigma(i), i^{\prime}\right), \quad i=1, \ldots, n-1, \quad i^{\prime}=1, \ldots, m, \\
& \left(n, i^{\prime}\right) \mapsto\left(n, i^{\prime}\right), \quad i^{\prime}=m+1, \ldots, m+n-3
\end{aligned}
$$

and $\sigma$ is the permutation in (3.2).

(iii) The number $N_{-}$of the negative monomials $\left[y_{\mathbf{i}}(u)\right]_{\mathbf{T}}$ for $(\mathbf{i}, u): \mathbf{p}_{+}$in the region $0 \leq u<$ $2(m n-m+n)$ is $n m^{2}-m^{2}+3 m n-8 m+2 n-6$.

\subsection{Remarks by referee}

The content of this subsection is based on the communication from the referee of this paper.

Recall that two quivers are said to be mutation-equivalent if one is obtained from the other by a sequence of mutations.

The following important observation was made by the referee.

Fact 1. The quiver $Q_{m, n}(\mathrm{SG})$ in Fig. 7 (Fig. 2 for $m=1$ ) is mutation-equivalent to the quiver of type $D_{m n-m+n}$, i.e., its underlying graph is the Dynkin diagram of type $D_{m n-m+n}$. Similarly, the quiver $Q_{m, n}(\mathrm{RSG})$ in Fig. 8 (Fig. 4 for $m=1$ ) is mutation-equivalent to the quiver of type $A_{m n-m+n-3}$. In other words, the cluster algebra $\mathcal{A}(B, x, y)$ with $B=B_{m, n}(\mathrm{SG})$ (resp. $\left.B=B_{m, n}(\mathrm{RSG})\right)$ is the cluster algebra of type $D_{m n-m+n}$ (resp. type $\left.A_{m n-m+n-3}\right)$.

For example, for $(m, n)=(1,7)$, this is seen as follows:

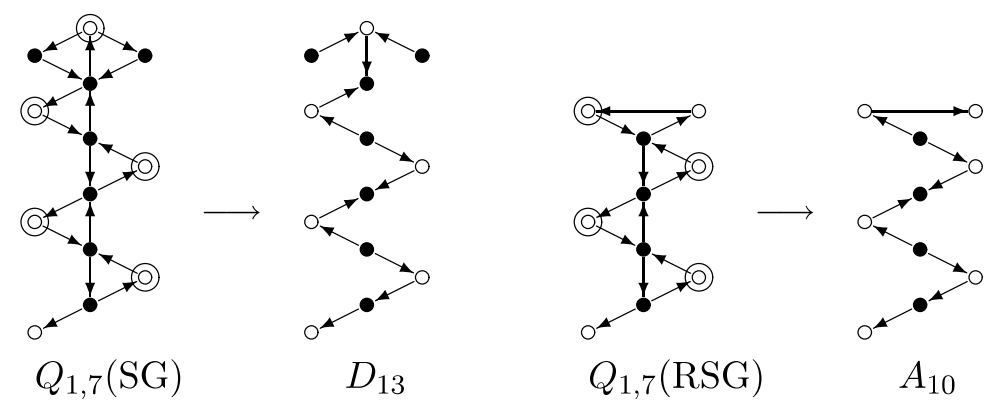

Here, we do mutations at the encircled vertices. For $m \geq 2$, the situation is a little more complicated and the examples for $(m, n)=(4,7)$ are given in Figs. 9 and 10.

The referee made a further observation on the periods in Theorems 7.3 and 7.9.

Fact 2. (a) Suppose that $m n-m+n$ is even. Then, the period $2(m n-m+n)$ in Theorems 7.3( $a$ ) coincides with $h\left(D_{m n-m+n}\right)+2$, which is the period of the Coxeter mutation sequence for the cluster algebra of type $D_{m n-m+n}$ studied by Fomin-Zelevinsky in $[8,9,10]$. Similarly, suppose that $m n-m+n$ is odd. Then, the period $4(m n-m+n)$ in Theorems $7.3(b)$ coincides with $2\left(h\left(D_{m n-m+n}\right)+2\right)$, which is the period of the Coxeter mutation sequence for the cluster algebra of type $D_{m n-m+n}$ in $[8,9,10]$. 

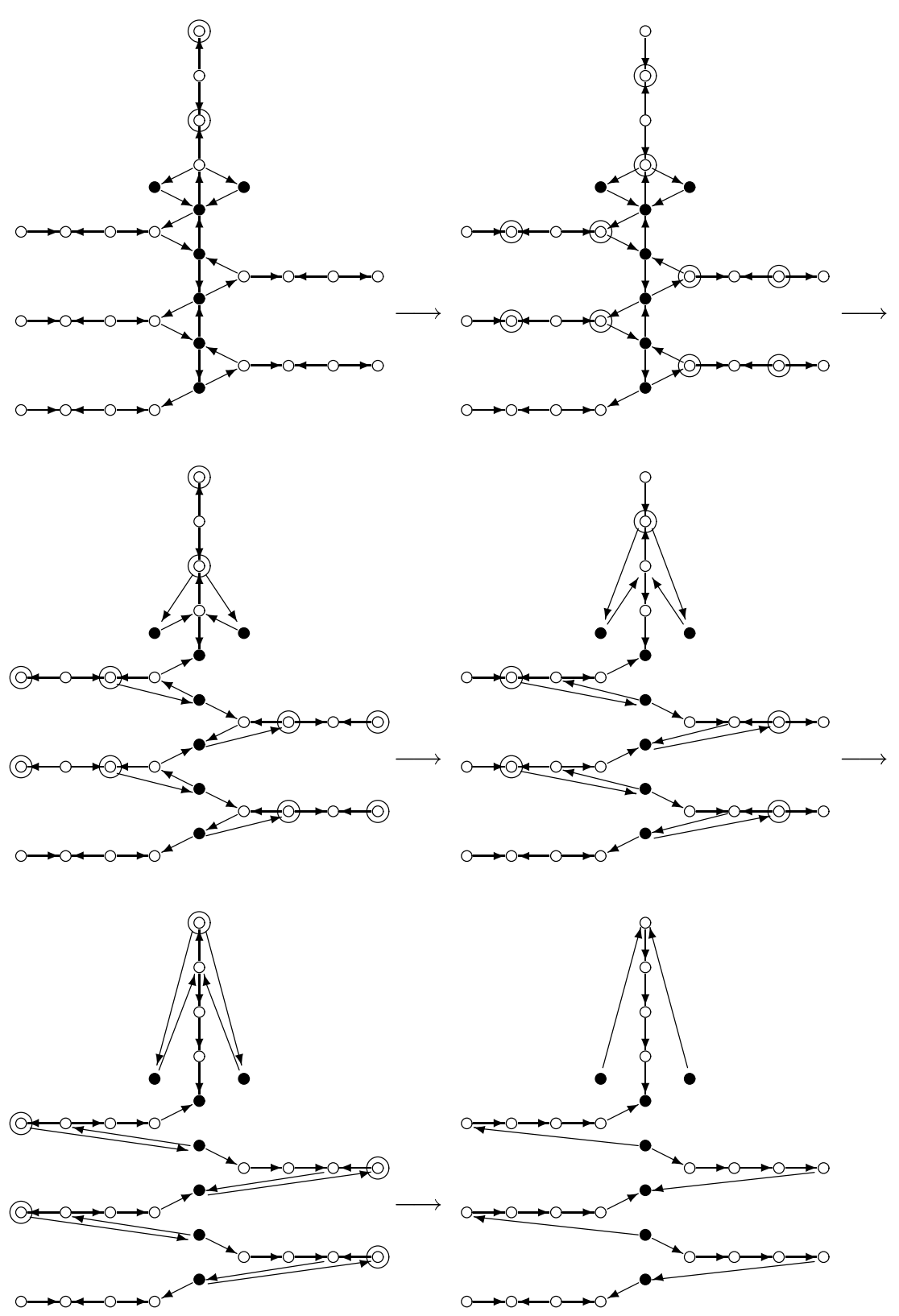

Figure 9. Sequence of mutations from $Q_{4,7}(\mathrm{SG})$ to the quiver of type $D_{31}$.

(b) The period $2(m n-m+n)$ in Theorems 7.9 coincides with $2\left(h\left(A_{m n-m+n-3}\right)+2\right)$, which is the period of the Coxeter mutation sequence for the cluster algebra of type $A_{m n-m+n-3}$ in $[8,9,10]$.

Facts 1 and 2 suggest that, even though the mutation sequences studied in this paper and the ones studied in $[8,9,10]$ are seemingly different, they may be related, or may be treated in a unified way. We leave this interesting question as an open problem.

\section{A Cluster algebras}

Here we collect basic definitions for cluster algebras to fix the convention and notation, mainly following [10]. For further necessary definitions and information for cluster algebras, see [10].

Let $I$ be a finite index set throughout the appendix. 

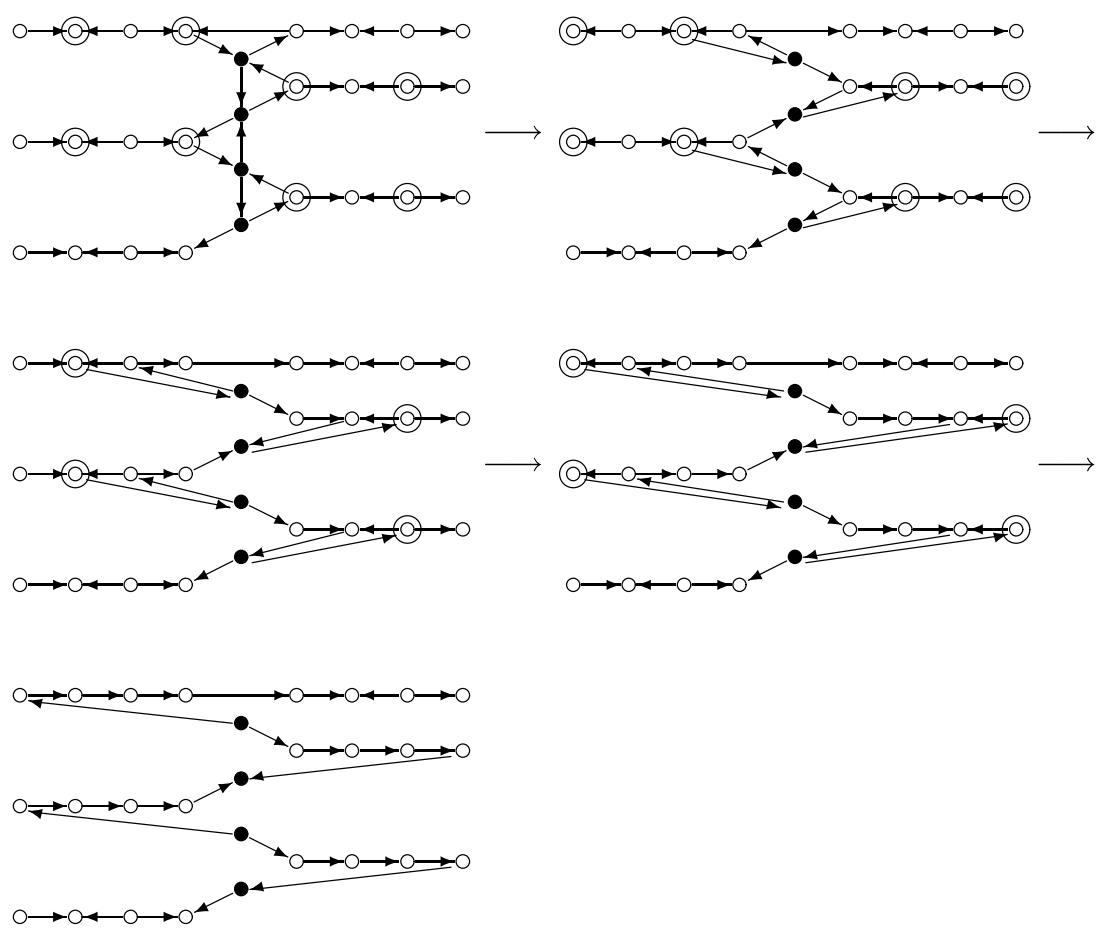

Figure 10. Sequence of mutations from $Q_{4,7}(\mathrm{RSG})$ to the quiver of type $A_{28}$.

(i) Semifield. A semifield $(\mathbb{P}, \oplus, \cdot)$ is an Abelian multiplicative group endowed with a binary operation of addition $\oplus$ which is commutative, associative, and distributive with respect to the multiplication in $\mathbb{P}$. The following three examples are important.

(a) Trivial semifield. The trivial semifield $\mathbf{1}=\{1\}$ consists of the multiplicative identity element 1 with $1 \oplus 1=1$.

(b) Universal semifield. For an I-tuple of variables $y=\left(y_{i}\right)_{i \in I}$, the universal semifield $\mathbb{P}_{\text {univ }}(y)$ consists of all the rational functions of the form $P(y) / Q(y)$ (subtraction-free rational expressions), where $P(y)$ and $Q(y)$ are the nonzero polynomials in $y_{i}$ 's with nonnegative integer coefficients. The multiplication and the addition are given by the usual ones of rational functions.

(c) Tropical semifield. For an $I$-tuple of variables $y=\left(y_{i}\right)_{i \in I}$, the tropical semifield $\mathbb{P}_{\text {trop }}(y)$ is the Abelian multiplicative group freely generated by the variables $y_{i}$ 's endowed with the addition $\oplus$

$$
\prod_{i} y_{i}^{a_{i}} \oplus \prod_{i} y_{i}^{b_{i}}=\prod_{i} y_{i}^{\min \left(a_{i}, b_{i}\right)} .
$$

(ii) Mutations of matrix and quiver. An integer matrix $B=\left(B_{i j}\right)_{i, j \in I}$ is skew-symmetrizable if there is a diagonal matrix $D=\operatorname{diag}\left(d_{i}\right)_{i \in I}$ with $d_{i} \in \mathbb{N}$ such that $D B$ is skew-symmetric. For a skew-symmetrizable matrix $B$ and $k \in I$, another matrix $B^{\prime}=\mu_{k}(B)$, called the mutation of $B$ at $k$, is defined by

$$
B_{i j}^{\prime}= \begin{cases}-B_{i j}, & i=k \text { or } j=k, \\ B_{i j}+\frac{1}{2}\left(\left|B_{i k}\right| B_{k j}+B_{i k}\left|B_{k j}\right|\right), & \text { otherwise. }\end{cases}
$$

The matrix $\mu_{k}(B)$ is also skew-symmetrizable.

It is standard to represent a skew-symmetric (integer) matrix $B=\left(B_{i j}\right)_{i, j \in I}$ by a quiver $Q$ without loops or 2 -cycles. The set of the vertices of $Q$ is given by $I$, and we put $B_{i j}$ arrows from $i$ 
to $j$ if $B_{i j}>0$. The mutation $Q^{\prime}=\mu_{k}(Q)$ of quiver $Q$ is given by the following rule: For each pair of an incoming arrow $i \rightarrow k$ and an outgoing arrow $k \rightarrow j$ in $Q$, add a new arrow $i \rightarrow j$. Then, remove a maximal set of pairwise disjoint 2-cycles. Finally, reverse all arrows incident with $k$.

(iii) Exchange relation of coefficient tuple. Let $\mathbb{P}$ be a given semifield. For an $I$-tuple $y=\left(y_{i}\right)_{i \in I}, y_{i} \in \mathbb{P}$ and $k \in I$, another $I$-tuple $y^{\prime}$ is defined by the exchange relation

$$
y_{i}^{\prime}= \begin{cases}y_{k}{ }^{-1}, & i=k, \\ y_{i}\left(\frac{y_{k}}{1 \oplus y_{k}}\right)^{B_{k i}}, & i \neq k, \quad B_{k i} \geq 0 \\ y_{i}\left(1 \oplus y_{k}\right)^{-B_{k i},} & i \neq k, \quad B_{k i} \leq 0 .\end{cases}
$$

(iv) Exchange relation of cluster. Let $\mathbb{Q P}$ be the quotient field of the group ring $\mathbb{Z P}$ of $\mathbb{P}$, and let $\mathbb{Q P}(z)$ be the rational function field of algebraically independent variables, say, $z=\left(z_{i}\right)_{i \in I}$ over $\mathbb{Q P}$. For an $I$-tuple $x=\left(x_{i}\right)_{i \in I}$ which is a free generating set of $\mathbb{Q P}(z)$ and $k \in I$, another $I$-tuple $x^{\prime}$ is defined by the exchange relation

$$
x_{i}^{\prime}= \begin{cases}x_{k}, & i \neq k, \\ y_{k} \prod_{j: B_{j k}>0} x_{j}^{B_{j k}}+\prod_{j: B_{j k}<0} x_{j}^{-B_{j k}} & \\ \left(1 \oplus y_{k}\right) x_{k} & i=k .\end{cases}
$$

(v) Seed mutation. For the above triplet $(B, x, y)$ in (ii)-(iv), which is called a seed, the mutation $\mu_{k}(B, x, y)=\left(B^{\prime}, x^{\prime}, y^{\prime}\right)$ at $k$ is defined by combining (A.2), (A.3), and (A.4).

(vi) Cluster algebra. Fix a semifield $\mathbb{P}$ and a seed (initial seed) $(B, x, y)$, where $x=\left(x_{i}\right)_{i \in I}$ are algebraically independent variables over $\mathbb{Q P}$, and $y=\left(y_{i}\right)_{i \in I}$ are elements in $\mathbb{P}$. Starting from $(B, x, y)$, iterate mutations and collect all the seeds $\left(B^{\prime}, x^{\prime}, y^{\prime}\right)$. We call $y^{\prime}$ and $y_{i}^{\prime}$ a coefficient tuple and a coefficient, respectively. We call $x^{\prime}$ and $x_{i}^{\prime} \in \mathbb{Q P}(x)$, a cluster and a cluster variable, respectively. The cluster algebra $\mathcal{A}(B, x, y)$ with coefficients in $\mathbb{P}$ is a $\mathbb{Z} \mathbb{P}$-subalgebra of the rational function field $\mathbb{Q P}(x)$ generated by all the cluster variables.

(vii) $\boldsymbol{F}$-polynomial. The cluster algebra $\mathcal{A}(B, x, y)$ with coefficients in the tropical semifield $\mathbb{P}_{\text {trop }}(y)$ is called the cluster algebra with the tropical coefficients (the principal coefficients in [10]). There, each cluster variable $x_{i}^{\prime}$ is an element in $\mathbb{Z}\left[x^{ \pm 1}, y\right]$. The $F$-polynomial $F_{i}^{\prime}(y) \in \mathbb{Z}[y]$ (for $x_{i}^{\prime}$ ) is defined as the specialization of $x_{i}^{\prime}$ with $x_{i}=1(i \in I)$.

\section{Acknowledgements}

It is our great pleasure to thank the anonymous referee who generously pointed out a crucial fact for the subject of the paper.

\section{References}

[1] Baxter R.J., Exactly solved models in statistical mechanics, Academic Press, Inc., London, 1982.

[2] Bazhanov V.V., Reshetikhin N., Restricted solid-on-solid models connected with simply laced algebras and conformal field theory, J. Phys. A: Math. Gen. 23 (1990), 1477-1492.

[3] Belavin A.A., Polyakov A.M., Zamolodchikov A.B., Infinite conformal symmetry in two-dimensional quantum field theory, Nuclear Phys. B 241 (1984), 333-380.

[4] Bernard D., LeClair A., Residual quantum symmetries of the restricted sine-Gordon theories, Nuclear Phys. B 340 (1990), 721-751.

[5] Chapoton F., Functional identities for the Rogers dilogarithm associated to cluster Y-systems, Bull. London Math. Soc. 37 (2005), 755-760. 
[6] Derksen H., Weyman J., Zelevinsky A., Quivers with potentials and their representations. II. Applications to cluster algebras, J. Amer. Math. Soc. 23 (2010), 749-790, arXiv:0904.0676.

[7] Fomin S., Zelevinsky A., Cluster algebras. I. Foundations, J. Amer. Math. Soc. 15 (2002), 497-529, math.RT/0104151.

[8] Fomin S., Zelevinsky A., Cluster algebras. II. Finite type classification, Invent. Math. 154 (2003), 63-121, math.RA/0208229.

[9] Fomin S., Zelevinsky A., Y-systems and generalized associahedra, Ann. of Math. (2) 158 (2003), 977-1018, hep-th/0111053.

[10] Fomin S., Zelevinsky A., Cluster algebras. IV. Coefficients, Compos. Math. 143 (2007), 112-164, math.RT/0602259.

[11] Frenkel E., Szenes A., Thermodynamic Bethe ansatz and dilogarithm identities. I, Math. Res. Lett. 2 (1995), 677-693, hep-th/9506215.

[12] Gliozzi F., Tateo R., ADE functional dilogarithm identities and integrable models, Phys. Lett. B 348 (1995), 677-693, hep-th/9411203.

[13] Inoue R., Iyama O., Keller B., Kuniba A., Nakanishi T., Periodicities of T and Y-systems, dilogarithm identities, and cluster algebras. I. Type $B_{r}$, arXiv:1001.1880.

[14] Inoue R., Iyama O., Keller B., Kuniba A., Nakanishi T., Periodicities of T and Y-systems, dilogarithm identities, and cluster algebras. II. Types $C_{r}, F_{4}$, and $G_{2}$, arXiv:1001.1881.

[15] Inoue R., Iyama O., Kuniba A., Nakanishi T., Suzuki J., Periodicities of T and Y-systems, Nagoya Math. J. 197 (2010), 59-174, arXiv:0812.0667.

[16] Keller B., Cluster algebras, quiver representations and triangulated categories, in Triangulated Categories, Editors T. Holm, P. Jørgensen and R. Rouquier, Lecture Note Series, Vol. 375, London Mathematical Society, Cambridge University Press, 2010, 76-160, arXiv:0807.1960.

[17] Keller B., The periodicity conjecture for pairs of Dynkin diagrams, arXiv:1001.1531.

[18] Kirillov A.N., Reshetikhin N.Y., Exact solution of the Heisenberg XXZ model of spin s, J. Soviet Math. 35 (1986), 2627-2643.

[19] Kirillov A.N., Reshetikhin N.Y., Representations of Yangians and multiplicities of the inclusion of the irreducible components of the tensor product of representations of simple Lie algebras, J. Soviet Math. 52 (1990), 3156-3164.

[20] Klassen T.R., Melzer E., Purely elastic scattering theories and their ultraviolet limits, Nuclear Phys. B 338 (1990), 485-528.

[21] Klümper A., Pearce P.A., Conformal weights of RSOS lattice models and their fusion hierarchies, Phys. A 183 (1992), 304-350.

[22] Kuniba A., Thermodynamics of the $U_{q}\left(X_{r}^{(1)}\right)$ Bethe ansatz system with $q$ a root of unity, Nuclear Phys. B 389 (1993), 209-244.

[23] Kuniba A., Nakanishi T., Spectra in conformal field theories from the Rogers dilogarithm, Modern Phys. Lett. A 7 (1992), 3487-3494, hep-th/9206034.

[24] Kuniba A., Nakanishi T., Suzuki J., Functional relations in solvable lattice models. I. Functional relations and representation theory, Internat. J. Modern Phys. A 9 (1994), 5215-5266, hep-th/9309137.

[25] Lewin L., Polylogarithms and associated functions, North-Holland, Amsterdam, 1981.

[26] Nakanishi T., Dilogarithm identities for conformal field theories and cluster algebras: simply laced case, Nagoya Math. J., to appear, arXiv:0909.5480.

[27] Onsager L., Crystal statistics. I. A two-dimensional model with an order disorder transition, Phys. Rev. 65 (1944), 117-149.

[28] Plamondon P., Cluster algebras via cluster categories with infinite-dimensional morphism spaces, arXiv:1004.0830.

[29] Plamondon P., Cluster characters for cluster categories with infinite-dimensional morphism spaces, arXiv:1002.4956.

[30] Ravanini R., Tateo R., Valleriani A., Dynkin TBA's, Internat. J. Modern Phys. A 8 (1993), 1707-1727, hep-th/9207040.

[31] Smirnov F.A., Reductions of the sine-Gordon model as a perturbation of minimal models of conformal field theory, Nuclear Phys. B 337 (1990), 156-180. 
[32] Tateo R., New functional dilogarithm identities and sine-Gordon Y-systems, Phys. Lett. B 355 (1995), 157-164, hep-th/9505022.

[33] Zamolodchikov A.B., Integrable field theory from conformal field theory, in Integrable Systems in Quantum Field Theory and Statistical Mechanics, Adv. Stud. Pure Math., Vol. 19, Academic Press, Boston, MA, 1989, 641-674.

[34] Zamolodchikov A.B., Thermodynamic Bethe ansatz in relativistic models: scaling 3-state Potts and LeeYang models, Nuclear Phys. B 342 (1990), 695-720.

[35] Zamolodchikov A.B., Zamolodchikov A.B., Factorized $S$-matrices in two dimensions as the exact solutions of certain relativistic quantum field theory models, Ann. Physics 120 (1979), 253-291.

[36] Zamolodchikov A.B., On the thermodynamic Bethe ansatz equations for reflectionless ADE scattering theories, Phys. Lett. B 253 (1991), 391-394. 